\title{
molecules
}

ISSN 1420-3049

www.mdpi.com/journal/molecules

Article

\section{Synthesis of Novel Highly Functionalized 4-Thiazolidinone Derivatives from 4-Phenyl-3-thiosemicarbazones}

\author{
Abdelmadjid Benmohammed ${ }^{1,2}$, Omar Khoumeri ${ }^{3}$, Ayada Djafri ${ }^{1}$, Thierry Terme $^{3}$ and \\ Patrice Vanelle $^{3, *}$
}

1 Laboratoire de Synthèse Organique Appliquée, BP 1524, El Menaouer, Département de Chimie, Faculté des Sciences Exactes et Appliquées, Université d'Oran, Oran 31000, Algeria;

E-Mails: medmadjid@yahoo.fr (A.B.); djafriayada@yahoo.fr (A.D.)

2 Département de Sciences et Techniques, Faculté des Sciences \& Technologie,

Université de Mascara, Mascara 29000, Algeria

3 Aix-Marseille Université, Institut de Chimie Radicalaire ICR, UMR CNRS 7273,

Laboratoire de Pharmaco-Chimie Radicalaire, Faculté de Pharmacie, 27 Boulevard Jean Moulin, Marseille cedex 05 13385, France; E-Mails: omar.khoumeri@univ-amu.fr (O.K.);

thierry.terme@univ-amu.fr (T.T.)

* Author to whom correspondence should be addressed; E-Mail: patrice.vanelle@univ-amu.fr; Tel.: +33-4-9183-5580; Fax: +33-4-9179-4677.

Received: 7 February 2014; in revised form: 27 February 2014 / Accepted: 4 March 2014 /

Published: 11 March 2014

\begin{abstract}
We present herein the synthesis in good yields of two series of highly functionalized thiazolidinone derivatives from the reactions of various 4-phenyl-3-thio-semicarbazones with ethyl 2-bromoacetate and diethyl acetylenedicarboxylate, respectively.
\end{abstract}

Keywords: 4-phenyl-3-thiosemicarbazones; thiazolidinones derivatives; ethyl 2-bromoacetate; diethyl acetylenedicarboxylate

\section{Introduction}

Thiosemicarbazones are a class of small molecules that have been evaluated over the last 50 years as antiviral [1] and as antitumoral agents [2-4], in addition to their antiparasitic and bacterial action against Trypanasoma cruzi [5-7] and Toxoplasma gondii and several bacterial strains [8]. Thiosemicarbazones have been used as intermediates for a great variety of heterocyclic products, such 
as thiazolidinones, thiohydantoins, thioxopyrimidinediones. It is reported that thiazolidinones exhibit antibacterial [9], antifungal [10], anticonvulsant [11], antitubercular [12], anti-inflammatory [13], antihistaminic [14,15], cardiovascular [16] and anti-HIV [17] activities. As part of our research program on new bioactive compounds [18-22], we report herein an efficient synthesis of some new highly functionalized thiazolidinones derived from 4-phenyl-3-thiosemicarbazones.

\section{Results and Discussion}

The starting materials, 4-phenyl-3-thiosemicarbazones $\mathbf{4 a - h}$, were synthesized in two steps. The first step was the preparation of 4-phenyl thiosemicarbazide (2) in 86\% yield from phenyl isothiocyanate (1) and hydrazine hydrate in ethanol at room temperature [23] (Scheme 1).

Scheme 1. Preparation of 4-phenyl-3-thiosemicarbazide (2).

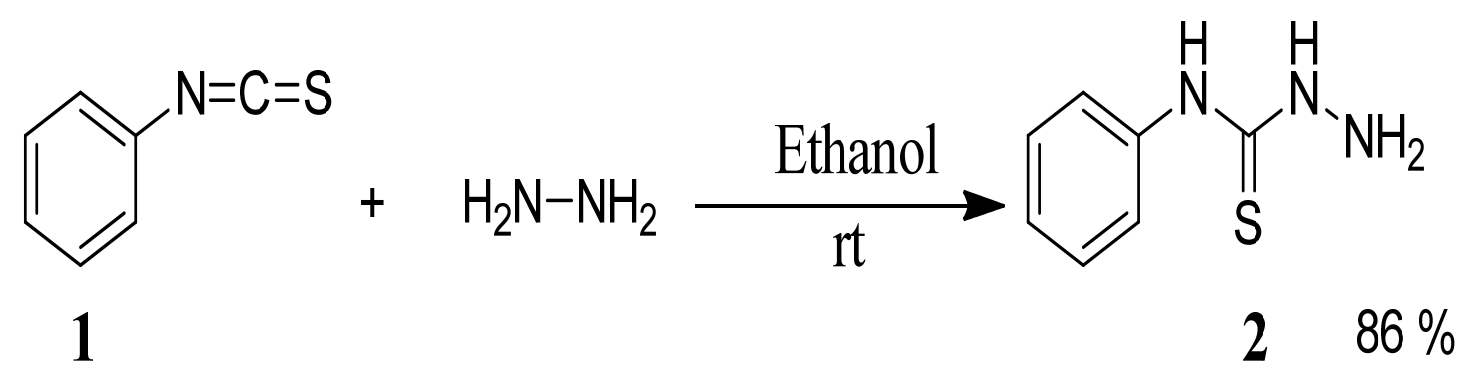

The reaction of 4-phenyl-3-thiosemicarbazide (2) with various aromatic aldehydes $\mathbf{3 a}-\mathbf{h}$ in the presence of few drops of acetic acid at $85^{\circ} \mathrm{C}$ for $1-3 \mathrm{~h}$, led to the corresponding 4-phenyl-3-thiosemicarbazone derivatives $\mathbf{4 a}-\mathbf{h}$ in good yields (70\%-93\%), as shown in Scheme 2 and Table 1.

Scheme 2. Preparation of 4-phenyl-3-thiosemicarbazones $\mathbf{4 a - h}$.<smiles>NNC(=S)Nc1ccccc1</smiles>

2<smiles>[R]C=O</smiles>

3a-h

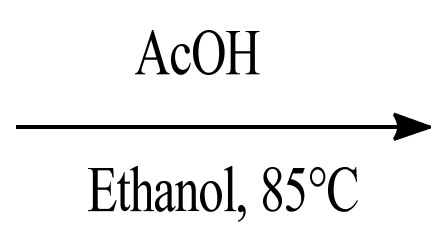

$1-3 \mathrm{~h}$<smiles>[R]/C=N\NC(=S)Nc1ccccc1</smiles>

4a-h $\quad 70-93 \%$

The most characteristic signals in the ${ }^{1} \mathrm{H}-\mathrm{NMR}$ spectrum of this family of thiosemicarbazones were those corresponding to the $\mathrm{CH}=\mathrm{N}$ and N-H protons. ${ }^{1} \mathrm{H}-\mathrm{NMR}$ studies showed the $\mathrm{CH}=\mathrm{N}$ protons in the 7.86-8.62 ppm range, whereas thiourea N-H protons are found in the 9.13-11.78 ppm interval for $\mathrm{N}-\mathrm{H}$ adjacent to the monosubstituted phenyl ring and for the $\mathrm{N}-\mathrm{H}$ adjacent to the $\mathrm{CH}=\mathrm{N}$ moiety, respectively. All of the synthesized compounds were in the E-configuration, which was confirmed using ${ }^{1} \mathrm{H}-\mathrm{NMR}$ spectroscopy, as the signal of the NH group was in the 9-12 ppm range, in comparison to the $Z$-isomer, which possesses a characteristic NH signal in the 14-15 ppm range [24]. 
Table 1. Reaction of $\mathbf{2}$ with various aromatic aldehydes $\mathbf{3 a}-\mathbf{h}$.

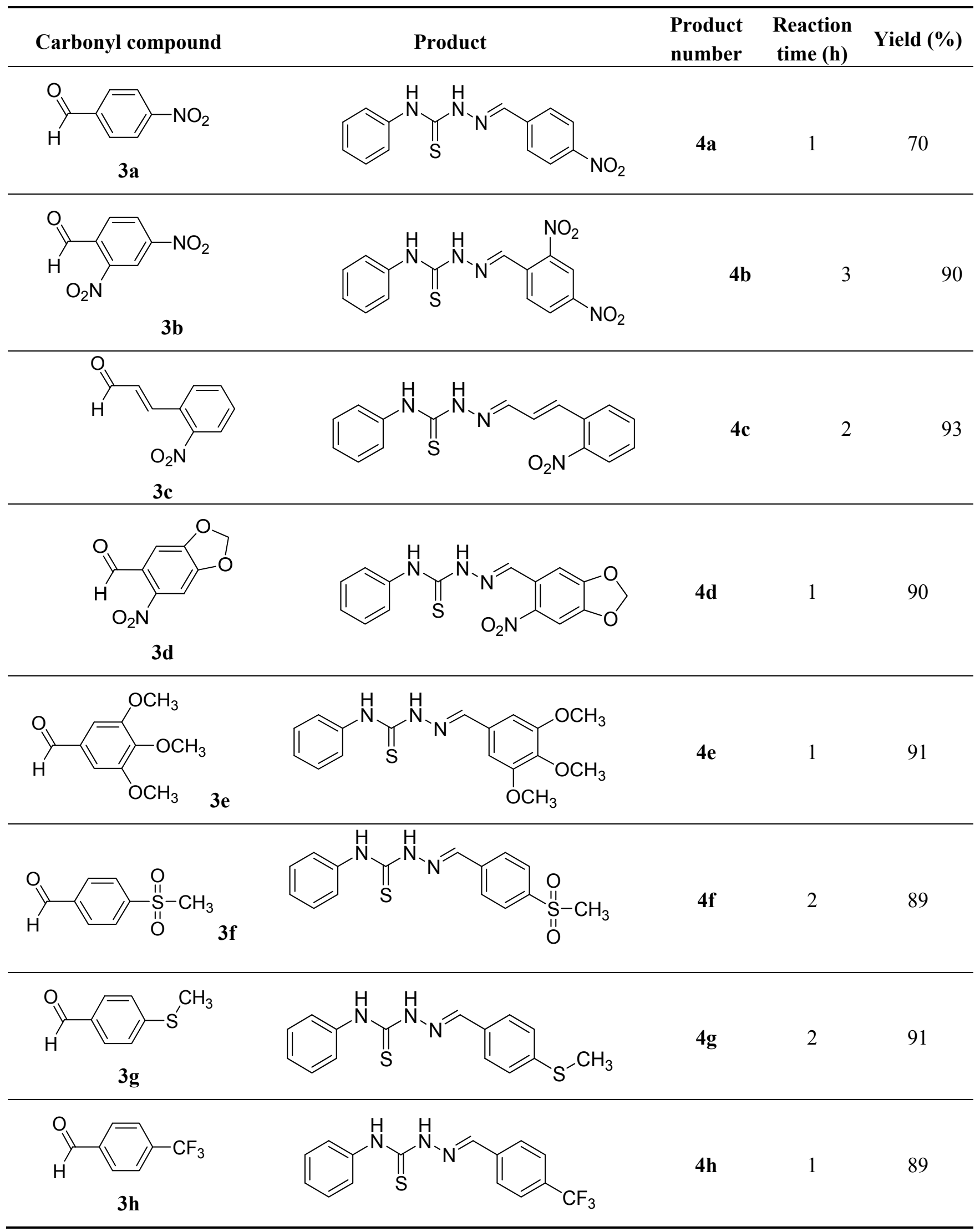


The reaction of various 4-phenyl thiosemicarbazones $\mathbf{4 a}-\mathbf{h}$ with ethyl 2-bromoacetate (5) as cyclizing reagent in boiling absolute ethanol containing three equivalents of anhydrous sodium acetate during 1-3 h, afforded to the thiazolidin-4-ones $\mathbf{6 a}-\mathbf{h}$ in good yields $(68 \%-91 \%)$ as shown in Scheme 3 and Table 2.

Scheme 3. Preparation of thiazolidinones $6 \mathbf{a}-\mathbf{h}$.<smiles>[R]C=NNC(=S)Nc1ccccc1</smiles>

Table 2. Reactions of $\mathbf{4 a}-\mathbf{h}$ with ethyl 2-bromoacetate (5).

Compound


Table 2. Cont.

Compound

The structures of all new compounds $\mathbf{6 a}-\mathbf{h}$ were established by analysis of their IR, ${ }^{1} \mathrm{H}-\mathrm{NMR}$ and ${ }^{13} \mathrm{C}$-NMR data. The IR spectra of the thiazolidin-4-ones $\mathbf{6 a}-\mathbf{h}$ showed absorption bands at about $1,734-1,716 \mathrm{~cm}^{-1}$ characteristic of (amide group) $\mathrm{C}=\mathrm{O}$ stretching vibrations. Further support was obtained from the ${ }^{1} \mathrm{H}$-NMR spectra, where it did not display signs of the 4-phenyl-3-thiosemicarbazone (NH) protons. On the other hand, the ${ }^{1} \mathrm{H}-\mathrm{NMR}$ spectra exhibited resonances assigned to the $\mathrm{SCH}_{2}$ group of the thiazolidine ring appearing as a singlet at $3.97-4.10 \mathrm{ppm}$ due to the methylene protons. The $\mathrm{CH}=\mathrm{N}$ protons in these structures were observed in the $7.67-8.57 \mathrm{ppm}$ region. The formation of thiazolidinones $\mathbf{6 a}-\mathbf{h}$ ocurred in two steps: the first step of this reaction is thought to be $S$-alkylation of thiosemicarbazide in its thiol form due to the sodium acetate used. Second step involved loss of ethanol to give the thiazolidin-4-one. The electronic and steric properties of the substituent at the 4-position of the thiosemicarbazones seems to be a determining factor for the formation of the thiazolidinone ring. Previous reports on these types of compounds reveal a small substituent such as phenyl or alkyl leads to a 4-thiazolidinone ring by loss of ethanol [25].

The next cyclization reaction of 4-phenyl-3-thiosemicarbazones derivatives $\mathbf{4 a}-\mathbf{h}$ was conducted

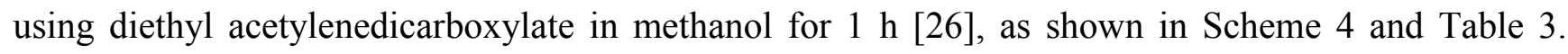
In this reaction both of the sulfur group and the amino group are capable of reacting with diethyl acetylenedicarboxylate. It was found that the 4-phenyl thiosemicarbazone derivatives $\mathbf{4} \mathbf{a}-\mathbf{h}$ reacted with diethyl acetylenedicarboxylate exclusively with the sulfur atom. In this reaction the intermediate 7 undergoes an intramolecular cyclization which leads to the compounds $\mathbf{8 a}-\mathbf{h}$. 
Scheme 4. Preparation of $\mathbf{8 a}-\mathbf{h}$ with 4-phenyl-3-thiosemicarbazones $\mathbf{4 a}-\mathbf{h}$ and diethyl acetylenedicarboxylate.

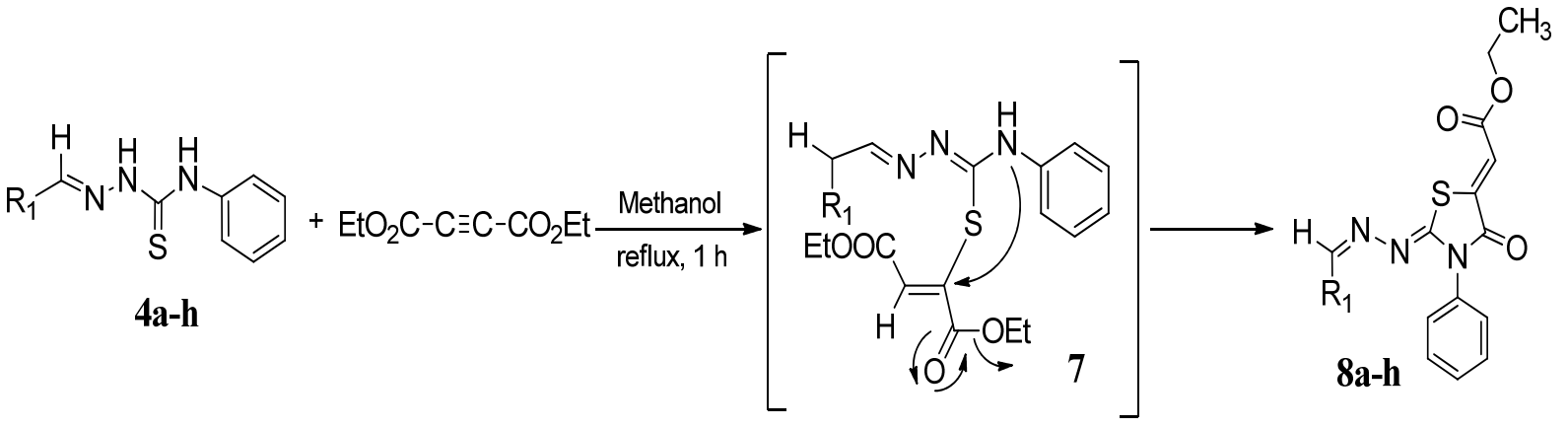

Table 3. Reactions of $\mathbf{4 a}-\mathbf{h}$ with diethyl acetylenedicarboxylate.

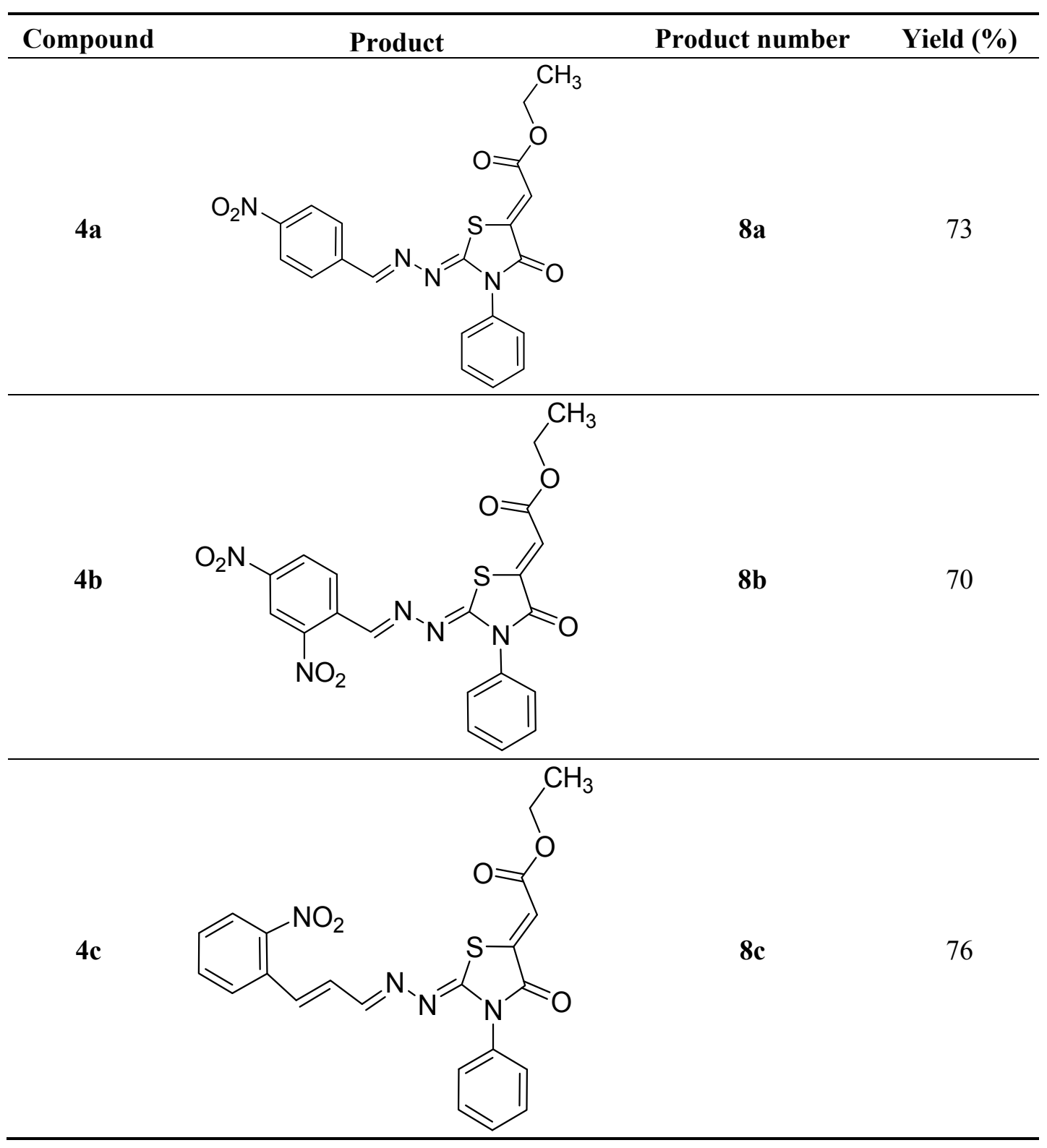


Table 3. Cont.

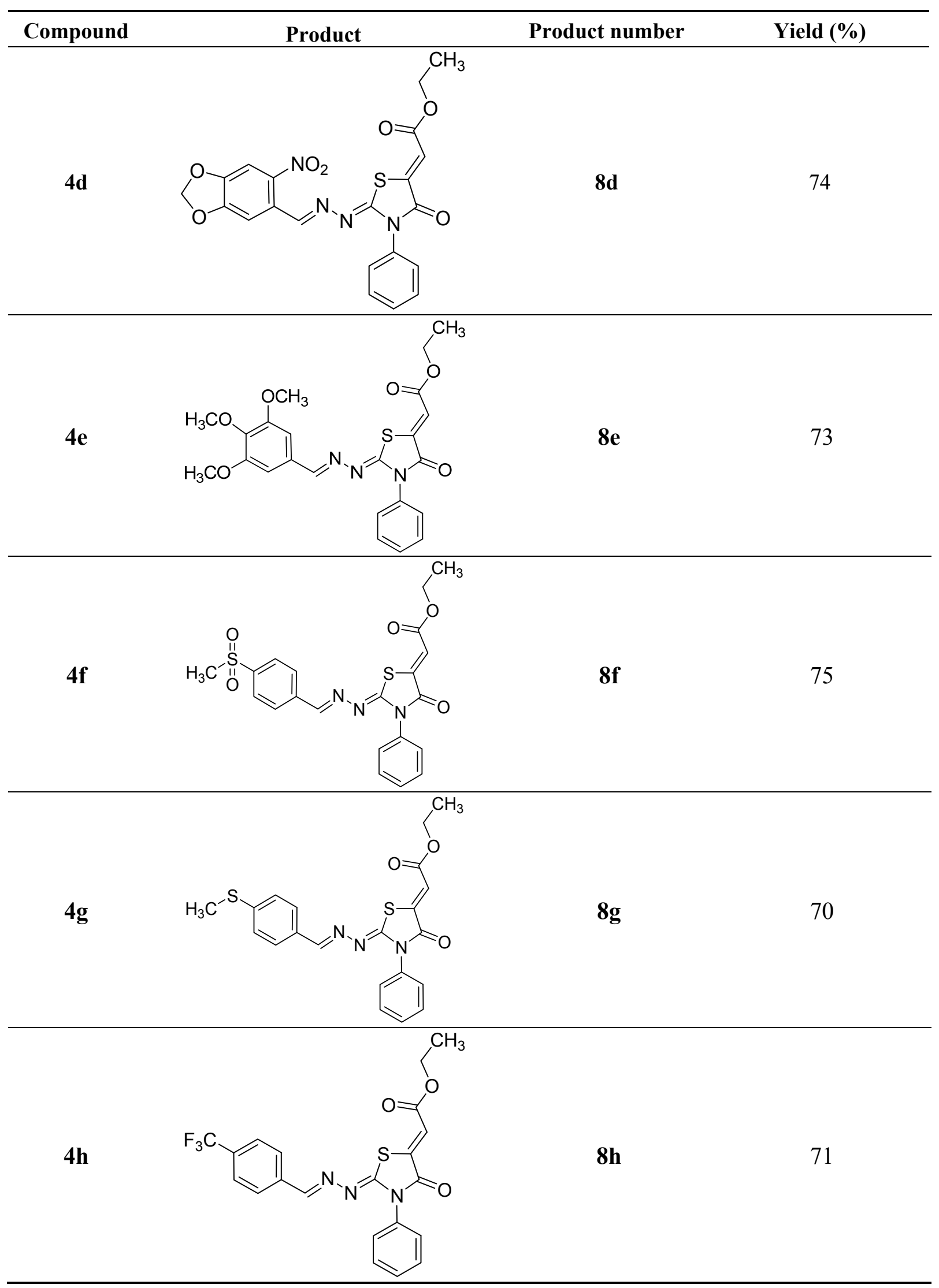

Although the two geometrical $E$ - or $Z$ - isomers of $\mathbf{8 a}-\mathbf{h}$ could be formed in almost equal amounts from the reaction of diethyl acetylenedicarboxylate with $\mathbf{4 a}-\mathbf{h},{ }^{1} \mathrm{H}-\mathrm{NMR}$ revealed the presence of only 
one singlet at $6.8 \mathrm{ppm}$ (vinyl proton) indicating that only one $E$ - or $Z$-isomer was formed. The structures of compound $\mathbf{8 b}$ and $\mathbf{8 g}$ obtained by X-ray structure analysis confirmed the Z-configuration for the double bond in the 5-position of the thiazolidin-4-ones (Figure 1) [27,28], probably due to the steric effect of the ester group.

Figure 1. ORTEP plots of $\mathbf{8 b}$ and $\mathbf{8 g}$.
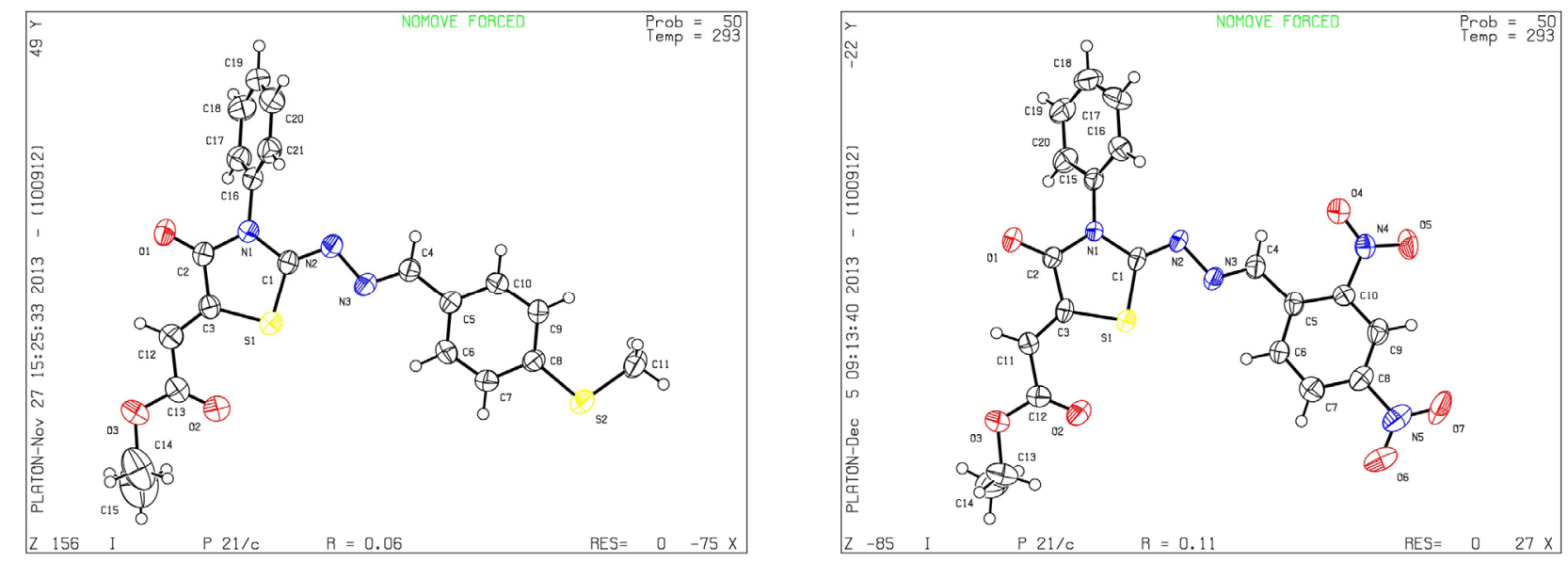

The chemical structures of the reaction products $8 \mathbf{a}-\mathbf{h}$ were confirmed by their IR, ${ }^{1} \mathrm{H}-\mathrm{NMR},{ }^{13} \mathrm{C}-\mathrm{NMR}$ spectra. The IR spectrum of compound 8a, for example, showed absorptions at $1730,1692 \mathrm{~cm}^{-1}$ due to the $\mathrm{C}=\mathrm{O}$ functions of the ester and cyclic amide, respectively. Similarly, bands at $1595-1622 \mathrm{~cm}^{-1}$ are due to the $\mathrm{C}=\mathrm{N}$ groups. The ${ }^{1} \mathrm{H}-\mathrm{NMR}$ spectrum of $\mathbf{8 a}$ showed a triplet at $\delta=1.28 \mathrm{ppm}$ and a quartet at $\delta=4.28 \mathrm{ppm}$ is due to the $\mathrm{COOCH}_{2} \mathrm{CH}_{3}$ protons. A singlet at $\delta=6.80 \mathrm{ppm}$ is due to $\mathrm{C}=\mathrm{CH}$. Aromatic protons appeared as a multiplet at $\delta=7.38-7.60 \mathrm{ppm}$.

\section{Experimental}

\subsection{General}

Melting points were determined on Büchi B-540 apparatus and are uncorrected. IR spectra were recorded as $\mathrm{KBr}$ pellets on a JASCO FT/IR4200 Fourier Transform infrared spectrometer and the reported wavenumbers are given in $\mathrm{cm}^{-1}$. Elemental analyses were carried out at the Spectropole, Faculté des Sciences site Saint-Jérome. ${ }^{1} \mathrm{H}-\mathrm{NMR}(200 \mathrm{MHz})$ and ${ }^{13} \mathrm{C}-\mathrm{NMR}(50 \mathrm{MHz})$ spectra were recorded on a Bruker ARX 200 spectrometer in $\mathrm{CDCl}_{3}$ or $\mathrm{D}_{2} \mathrm{O}$ at the Service Inter-Universitaire de RMN de la Faculté de Pharmacie de Marseille. The ${ }^{1} \mathrm{H}-\mathrm{NMR}$ chemical shifts were reported as parts per million downfield from tetramethylsilane $\left(\mathrm{Me}_{4} \mathrm{Si}\right)$, and the ${ }^{13} \mathrm{C}-\mathrm{NMR}$ chemical shifts were referenced to the solvent peaks: $\mathrm{CDCl}_{3}(76.9 \mathrm{ppm})$ or DMSO-d 6 (39.6 ppm). Silica gel 60 (Merck, 230-400 mesh) was used for column chromatography: Thin-layer chromatography was performed with silica gel Merck 60F-254 (0.25 mm layer thickness). 


\subsection{General Procedure for the Preparation of Compounds $\mathbf{4 a}-\mathbf{h}$}

To a solution of 4-phenylthiosemicarbazide (2, $1 \mathrm{~g}, 6 \mathrm{mmol}, 1 \mathrm{eq})$ in ethanol (33 $\mathrm{mL})$ were added the benzaldehyde derivative $(6.3 \mathrm{mmol}, 1.05 \mathrm{eq})$ and acetic acid $(0.50 \mathrm{~mL})$. The mixture was stirred under reflux for $1-3 \mathrm{~h}$ and then cooled to room temperature. After, the solid separated was filtered and recrystallized from ethanol-DMF (3:1) to give compounds $\mathbf{4 a}-\mathbf{h}$.

(E)-2-(4-Nitrobenzylidene)-N-phenylhydrazinecarbothioamide (4a): yellow solid; mp: $234{ }^{\circ} \mathrm{C}$; IR $(\mathrm{KBr}) v \max / \mathrm{cm}^{-1}$ : 3316 and $3138(\mathrm{NH}), 1543(\mathrm{C}=\mathrm{N}), 1292(\mathrm{C}=\mathrm{S}) ;{ }^{1} \mathrm{H}-\mathrm{NMR}\left(\mathrm{DMSO}-\mathrm{d}_{6}\right): \delta 10.32$ $(\mathrm{s}, 1 \mathrm{H}, \mathrm{NH}), 8.24(\mathrm{~d}, 2 \mathrm{H}, J=9.2 \mathrm{~Hz}, \mathrm{Ar}-\mathrm{H}), 8.22(\mathrm{~s}, 1 \mathrm{H}, \mathrm{CH}=\mathrm{N}), 8.17(\mathrm{~d}, 2 \mathrm{H}, J=9.2 \mathrm{~Hz}, \mathrm{Ar}-\mathrm{H}), 7.52$ (d, $2 \mathrm{H}, J=7.9 \mathrm{~Hz}, \mathrm{Ar}-\mathrm{H}), 7.38$ (dd, $2 \mathrm{H}, J=7.9 \mathrm{~Hz}, J=7.2 \mathrm{~Hz}, \mathrm{Ar}-\mathrm{H}), 7.22$ ( t $, 1 \mathrm{H} J=7.2 \mathrm{~Hz}, \mathrm{Ar}-\mathrm{H}$ ); ${ }^{13} \mathrm{C}-\mathrm{NMR}\left(\mathrm{DMSO}_{\mathrm{d}}\right.$ ): $\delta 177.0(\mathrm{C}), 148.1(\mathrm{C}), 141.0(\mathrm{C}), 140.6(\mathrm{CH}), 139.4(\mathrm{C}), 128.9(2 \mathrm{CH}), 128.6$ $(2 \mathrm{CH}), 126.7(2 \mathrm{CH}), 126.1(\mathrm{CH}), 124.4(2 \mathrm{CH})$; Anal. Calcd for $\mathrm{C}_{14} \mathrm{H}_{12} \mathrm{~N}_{4} \mathrm{O}_{2} \mathrm{~S}: \mathrm{C}, 55.99 ; \mathrm{H}, 4.03 ; \mathrm{N}$, 18.65; S, 10.68. Found: C, 55.98; H, 4.06; N, 18.49; S, 10.68.

(E)-2-(2,4-Dinitrobenzylidene)-N-phenylhydrazinecarbothioamide (4b): yellow solid; mp: $219{ }^{\circ} \mathrm{C}$; IR $(\mathrm{KBr}) v_{\max } / \mathrm{cm}^{-1}: 3316$ and $3138(\mathrm{NH}), 1543(\mathrm{C}=\mathrm{N}), 1292(\mathrm{C}=\mathrm{S}) ;{ }^{1} \mathrm{H}-\mathrm{NMR}\left(\mathrm{DMSO}-\mathrm{d}_{6}\right): \delta 10.40$ (s, $1 \mathrm{H}, \mathrm{NH}), 8.87$ (d, $1 \mathrm{H}, J=8.8 \mathrm{~Hz}, \operatorname{Ar}-\mathrm{H}), 8.75$ (d, 1H, $J=2.2 \mathrm{~Hz}, \mathrm{Ar}-\mathrm{H}), 8.62(\mathrm{~s}, 1 \mathrm{H}, \mathrm{CH}=\mathrm{N})$, $8.45(\mathrm{dd}, 1 \mathrm{H}, J=8.8 \mathrm{~Hz}, J=2.2 \mathrm{~Hz}, \mathrm{Ar}-\mathrm{H}), 7.52(\mathrm{~d}, 2 \mathrm{H}, J=7.7 \mathrm{~Hz}, \mathrm{Ar}-\mathrm{H}), 7.39$ (dd, $2 \mathrm{H}, J=7.7 \mathrm{~Hz}$, $J=7.3 \mathrm{~Hz}, \mathrm{Ar}-\mathrm{H}), 7.24(\mathrm{t}, 1 \mathrm{H}, J=7.3 \mathrm{~Hz}, \mathrm{Ar}-\mathrm{H}) ;{ }^{13} \mathrm{C}-\mathrm{NMR}\left(\mathrm{DMSO}-\mathrm{d}_{6}\right): \delta 177.2(\mathrm{C}), 148.3(\mathrm{C}), 147.5$ (C), $139.2(\mathrm{C}), 136.4(\mathrm{CH}), 134.6(\mathrm{C}), 130.3(\mathrm{CH}), 128.7(2 \mathrm{CH}), 127.4(\mathrm{CH}), 126.6(2 \mathrm{CH}), 126.3$ (CH), $120.8(\mathrm{CH})$; Anal. Calcd for $\mathrm{C}_{14} \mathrm{H}_{11} \mathrm{~N}_{5} \mathrm{O}_{4} \mathrm{~S}$ : C, 48.69; H, 3.21; N, 20.28; S, 9.29. Found: C, 48.83; H, 3.22; N, 20.08; S, 9.05 .

(E)-2-((E)-3-(2-Nitrophenyl)allylidene)-N-phenylhydrazinecarbothioamide (4c): brown solid; mp: $185{ }^{\circ} \mathrm{C}$; IR $(\mathrm{KBr}) v_{\max } / \mathrm{cm}^{-1}: 3226$ and $3151(\mathrm{NH}), 1540(\mathrm{C}=\mathrm{N}), 1247(\mathrm{C}=\mathrm{S}) ;{ }^{1} \mathrm{H}-\mathrm{NMR}\left(\mathrm{CDCl}_{3}\right): \delta 9.67(\mathrm{~s}, 1 \mathrm{H}$, $\mathrm{NH}), 9.14$ (s, 1H, NH), 8.02 (dd, 1H, $J=8.2 \mathrm{~Hz}, J=1.2 \mathrm{~Hz}, \mathrm{Ar}-\mathrm{H}), 7.76-7.59$ (m, 5H, Ar-H), 7.52$7.37(\mathrm{~m}, 4 \mathrm{H}, \mathrm{Ar}-\mathrm{H}), 7.25-7.29(\mathrm{~m}, 1 \mathrm{H}, \mathrm{Ar}-\mathrm{H}), 6.87(\mathrm{dd}, 1 \mathrm{H}, J=15.9 \mathrm{~Hz}, J=9.2 \mathrm{~Hz}, \mathrm{CH})$; ${ }^{13} \mathrm{C}-\mathrm{NMR}\left(\mathrm{CDCl}_{3}\right): \delta 175.6(\mathrm{C}), 147.8(\mathrm{C}), 143.3(\mathrm{CH}), 137.7(\mathrm{C}), 134.8(\mathrm{CH}), 133.3(\mathrm{CH}), 131.3(\mathrm{C})$, 129.4 (CH), $129.0(\mathrm{CH}), 128.8(2 \mathrm{CH}), 128.2(\mathrm{CH}), 126.2(\mathrm{CH}), 125.0(\mathrm{CH}), 124.2(2 \mathrm{CH})$; Anal. Calcd for $\mathrm{C}_{16} \mathrm{H}_{14} \mathrm{~N}_{4} \mathrm{O}_{2} \mathrm{~S}$ : C, 58.88; H, 4.32; N, 17.17; S, 9.82. Found: C, 59.13; H, 4.40; N, 17.07; S, 9.78.

(E)-2-((6-Nitrobenzo[d][1,3]dioxol-5-yl)methylene)-N-phenylhydrazinecarbothioamide (4d): yellow solid; mp: $231{ }^{\circ} \mathrm{C}$; IR (KBr) $v_{\max } / \mathrm{cm}^{-1}$ : 3302 and $3283(\mathrm{NH}), 1547(\mathrm{C}=\mathrm{N}), 1268(\mathrm{C}=\mathrm{S})$; ${ }^{1} \mathrm{H}-\mathrm{NMR}$ $\left(\mathrm{DMSO}_{-} \mathrm{d}_{6}\right): \delta 10.21(\mathrm{~s}, 1 \mathrm{H}, \mathrm{NH}), 8.58(\mathrm{~s}, 1 \mathrm{H}, \operatorname{Ar}-\mathrm{H}), 8.17(\mathrm{~s}, 1 \mathrm{H}, \mathrm{CH}=\mathrm{N}), 7.61(\mathrm{~s}, 1 \mathrm{H}, \operatorname{Ar}-\mathrm{H})$, 7.50 (dd, 2H, $J=7.8 \mathrm{~Hz}, J=1.2 \mathrm{~Hz}, \mathrm{Ar}-\mathrm{H}), 7.37$ (dd, 2H, $J=7.8 \mathrm{~Hz}, J=7.2 \mathrm{~Hz}, \mathrm{Ar}-\mathrm{H}), 7.21$ (td, $1 \mathrm{H}, J=7.2 \mathrm{~Hz}, J=1.2 \mathrm{~Hz}, \mathrm{Ar}-\mathrm{H}), 6.25\left(\mathrm{~s}, 2 \mathrm{H}, \mathrm{CH}_{2}\right) ;{ }^{13} \mathrm{C}-\mathrm{NMR}\left(\mathrm{DMSO}_{6}\right): \delta 176.9(\mathrm{C}), 152.2(\mathrm{C})$, $149.2(\mathrm{C}), 143.9(\mathrm{C}), 139.4(\mathrm{C}), 138.5(\mathrm{CH}), 128.6(2 \mathrm{CH}), 126.94(2 \mathrm{CH}), 126.1(\mathrm{CH}), 125.9(\mathrm{C}), 106.6$ $(\mathrm{CH}), 105.3(\mathrm{CH}), 104.1\left(\mathrm{CH}_{2}\right)$; Anal. Calcd for $\mathrm{C}_{15} \mathrm{H}_{12} \mathrm{~N}_{4} \mathrm{O}_{4} \mathrm{~S}: \mathrm{C}, 52.32 ; \mathrm{H}, 3.51 ; \mathrm{N}, 16.27 ; \mathrm{S}, 9.31$. Found: C, 52.35; H, 3.46; N, 16.01; S, 9.20.

(E)-N-Phenyl-2-(3,4,5-trimethoxybenzylidene)hydrazinecarbothioamide (4e): white solide; mp: $161{ }^{\circ} \mathrm{C}$; IR $(\mathrm{KBr}) v_{\max } / \mathrm{cm}^{-1}: 3291$ and $3134(\mathrm{NH}), 1557(\mathrm{C}=\mathrm{N}), 1262(\mathrm{C}=\mathrm{S}) ;{ }^{1} \mathrm{H}-\mathrm{NMR}\left(\mathrm{CDCl}_{3}\right)$ : 
$\delta 10.36(\mathrm{~s}, 1 \mathrm{H}, \mathrm{NH}), 9.14(\mathrm{~s}, 1 \mathrm{H}, \mathrm{NH}), 7.93(\mathrm{~s}, 1 \mathrm{H}, \mathrm{CH}=\mathrm{N}), 7.63(\mathrm{~d}, 2 \mathrm{H}, J=7.8 \mathrm{~Hz}, \mathrm{Ar}-\mathrm{H}), 7.44$ (dd, 2H, $J=7.8 \mathrm{~Hz}, J=7.2 \mathrm{~Hz}, \operatorname{Ar}-\mathrm{H}), 7.30$ (t, 1H, $J=7.2 \mathrm{~Hz}, \operatorname{Ar}-\mathrm{H}$ ), 6.90 (s, 2H, Ar-H ), 3.90 $\left(\mathrm{s}, 3 \mathrm{H}, \mathrm{OCH}_{3}\right), 3.91\left(\mathrm{~s}, 6 \mathrm{H}, \mathrm{OCH}_{3}\right) ;{ }^{13} \mathrm{C}-\mathrm{NMR}\left(\mathrm{CDCl}_{3}\right): \delta 175.6(\mathrm{C}), 153.6(2 \mathrm{C}), 143.7(\mathrm{CH}), 140.5$ (C), $137.7(\mathrm{C}), 129.0(2 \mathrm{CH}), 128.4(\mathrm{C}), 126.6(\mathrm{CH}), 125.2(2 \mathrm{CH}), 104.7(2 \mathrm{CH}), 61.1\left(\mathrm{CH}_{3}\right), 56.3$ $\left(2 \mathrm{CH}_{3}\right)$; Anal. Calcd for $\mathrm{C}_{17} \mathrm{H}_{19} \mathrm{~N}_{3} \mathrm{O}_{3} \mathrm{~S}$ : C, 59.11; H, 5.54; N, 12.17; S, 9.28. Found: C, 59.25; H, 5.65; N, 12.06; S, 9.19.

(E)-2-(4-(Methylsulfonyl)benzylidene)-N-phenylhydrazinecarbothioamide (4f): yellow solid; mp: $225{ }^{\circ} \mathrm{C}$; IR (KBr) $v_{\max } / \mathrm{cm}^{-1}$ : 3325 and $3150(\mathrm{NH}), 1542(\mathrm{C}=\mathrm{N}), 1267(\mathrm{C}=\mathrm{S}) ;{ }^{1} \mathrm{H}-\mathrm{NMR}\left(\mathrm{DMSO}-\mathrm{d}_{6}\right)$ : $\delta 10.30(\mathrm{~s}, 1 \mathrm{H}, \mathrm{NH}), 8.22(\mathrm{~s}, 1 \mathrm{H}, \mathrm{CH}=\mathrm{N}), 8.19(\mathrm{~d}, 2 \mathrm{H}, J=8.4 \mathrm{~Hz}, \mathrm{Ar}-\mathrm{H}), 7.95$ (d, 2H, $J=8.4 \mathrm{~Hz}$, Ar-H), $7.55(\mathrm{~d}, 2 \mathrm{H}, J=7.60 \mathrm{~Hz}, \mathrm{Ar}-\mathrm{H}), 7.39$ (dd, 2H, $J=7.2 \mathrm{~Hz}, J=7.60 \mathrm{~Hz}, \mathrm{Ar}-\mathrm{H}), 7.23(\mathrm{t}, 1 \mathrm{H}$, $J=7.2 \mathrm{~Hz}, \mathrm{Ar}-\mathrm{H}), 3.26\left(\mathrm{~s}, 3 \mathrm{H}, \mathrm{CH}_{3}\right) ;{ }^{13} \mathrm{C}-\mathrm{NMR}\left(\mathrm{DMSO}_{-} \mathrm{d}_{6}\right): \delta 177.0(\mathrm{C}), 141.7(\mathrm{C}), 141.2(\mathrm{CH})$, $139.44(2 \mathrm{C}), 128.7(2 \mathrm{CH}), 128.6(2 \mathrm{CH}), 127.7(2 \mathrm{CH}), 126.7(2 \mathrm{CH}), 126.10(\mathrm{CH}), 43.9\left(\mathrm{CH}_{3}\right)$; Anal. Calcd for $\mathrm{C}_{15} \mathrm{H}_{15} \mathrm{~N}_{3} \mathrm{O}_{2} \mathrm{~S}_{2}$ : C, 54.03; H, 4.53; N, 12.60; S, 19.23. Found: C, 54.11; H, 4.55; N, $12.45 ; \mathrm{S}, 19.21$.

(E)-2-(4-(Methylthio)benzylidene)-N-phenylhydrazinecarbothioamide (4g): yellow solid; mp: $178{ }^{\circ} \mathrm{C}$; IR $(\mathrm{KBr}) v_{\max } / \mathrm{cm}^{-1}: 3343$ and $3152(\mathrm{NH}), 1541(\mathrm{C}=\mathrm{N}), 1271(\mathrm{C}=\mathrm{S}) ;{ }^{1} \mathrm{H}-\mathrm{NMR}\left(\mathrm{CDCl}_{3}\right): \delta 11.78(\mathrm{~s}, 1 \mathrm{H}$, $\mathrm{NH}), 10.08(\mathrm{~s}, 1 \mathrm{H}, \mathrm{NH}), 8.09(\mathrm{~s}, 1 \mathrm{H}, \mathrm{CH}=\mathrm{N}), 7.82(\mathrm{~d}, 2 \mathrm{H}, J=8.4 \mathrm{~Hz}, \mathrm{Ar}-\mathrm{H}), 7.54(\mathrm{~d}, 2 \mathrm{H}, J=8.0 \mathrm{~Hz}$, Ar-H), 7.35 (dd, 2H, $J=7.3 \mathrm{~Hz}, J=8.0 \mathrm{~Hz}, \operatorname{Ar}-\mathrm{H}), 7.27$ (d, $J=8.4 \mathrm{~Hz}, 2 \mathrm{H}, \mathrm{Ar}-\mathrm{H}), 7.18$ (dd, 1H, $J=7.3 \mathrm{~Hz}, \mathrm{Ar}-\mathrm{H}),{ }^{13} \mathrm{C}-\mathrm{NMR}\left(\mathrm{DMSO}_{6}\right)$ : $\delta 175.4(\mathrm{C}), 143.1(\mathrm{CH}), 142.5(\mathrm{C}), 137.8(\mathrm{C}), 129.6(\mathrm{C})$, $128.9(2 \mathrm{CH}), 127.8(2 \mathrm{CH}), 126.3(\mathrm{CH}), 125.8(2 \mathrm{CH}), 124.8(2 \mathrm{CH}), 15.1\left(\mathrm{CH}_{3}\right)$; Anal. Calcd for $\mathrm{C}_{15} \mathrm{H}_{15} \mathrm{~N}_{3} \mathrm{O}_{2} \mathrm{~S}_{2}$ : C, 59.77; H, 5.02; N, 13.94; S, 21.28. Found: C, 59.68; H, 5.04; N, 13.78; S, 21.33.

(E)-N-Phenyl-2-(4-(trifluoromethyl)benzylidene)hydrazinecarbothioamide (4h): white solid; mp: $195{ }^{\circ} \mathrm{C}$; IR $(\mathrm{KBr}) v_{\max } / \mathrm{cm}^{-1}: 3350$ and $3138(\mathrm{NH}), 1542(\mathrm{C}=\mathrm{N}), 1267(\mathrm{C}=\mathrm{S}) ;{ }^{1} \mathrm{H}-\mathrm{NMR}\left(\mathrm{CDCl}_{3}\right)$ : $\delta 10.36(\mathrm{~s}, 1 \mathrm{H}, \mathrm{NH}), 9.18(\mathrm{~s}, 1 \mathrm{H}, \mathrm{NH}), 8.03(\mathrm{~s}, 1 \mathrm{H}, \mathrm{CH}=\mathrm{N}), 7.85(\mathrm{~d}, 2 \mathrm{H}, J=8.1 \mathrm{~Hz}, \mathrm{Ar}-\mathrm{H}), 7.69-7.51$ (m, 4H, Ar-H), 7.43 (d, 2H, $J=7.4 \mathrm{~Hz}, \mathrm{Ar}-\mathrm{H}), 7.29$ (t, $1 \mathrm{H}, J=7.3 \mathrm{~Hz}, \mathrm{Ar}-\mathrm{H}) ;{ }^{13} \mathrm{C}-\mathrm{NMR}\left(\mathrm{CDCl}_{3}\right)$ : $\delta 176.0(\mathrm{C}), 141.4(\mathrm{CH}), 137.5(\mathrm{C}), 133.9(\mathrm{C}), 131.5$ (q, $J=32.9 \mathrm{~Hz}, \mathrm{C}), 129.5(\mathrm{CH}), 128.9(3 \mathrm{CH})$, 127.0 (q, $J=3.6 \mathrm{~Hz}, \mathrm{CH}), 126.6(\mathrm{CH}), 124.9(2 \mathrm{CH}), 123.9$ (q, $J=3.6 \mathrm{~Hz}, \mathrm{CH}), 123.7$ (q, $J=272.6 \mathrm{~Hz}$, C); Anal. Calcd for $\mathrm{C}_{15} \mathrm{H}_{12} \mathrm{~F}_{3} \mathrm{~N}_{3} \mathrm{~S}$ : C, 55.72; H, 3.74; N, 13.00; S, 9.92. Found: C, 55.79; H, 3.66; N, 12.86; S, 9.81 .

\subsection{General Procedure for the Preparation of Compounds $\mathbf{6 a}-\mathbf{h}$}

A mixture of compound $\mathbf{4 a}-\mathbf{h}(1.5 \mathrm{mmol}, 1 \mathrm{eq})$, ethyl 2-bromoacetate $(0.24 \mathrm{~mL}, 1.5 \mathrm{mmol})$ and anhydrous sodium acetate $(0.37 \mathrm{~g}, 4.5 \mathrm{mmol}, 3 \mathrm{eq})$ in ethanol $(30 \mathrm{~mL})$ was stirred until reflux; the mixture was stirred under the same conditions till the completion of the reaction $(1-3 \mathrm{~h})$. The reaction mixture was left to cool, poured into ice cold water, and the separated solid was filtered, washed with water and recrystallized from a mixture of ethanol-DMF $(3: 1)$.

2-((4-Nitrobenzylidene)hydrazono)-3-phenylthiazolidin-4-one (6a): yellow solid; $\mathrm{mp}: 258{ }^{\circ} \mathrm{C}$; IR $(\mathrm{KBr}) v_{\max } / \mathrm{cm}^{-1}: 1716(\mathrm{C}=\mathrm{O}), 1662(\mathrm{C}=\mathrm{N}) ;{ }^{1} \mathrm{H}-\mathrm{NMR}\left(\mathrm{CDCl}_{3}\right): \delta 8.34(\mathrm{~s}, 1 \mathrm{H}, \mathrm{CH}=\mathrm{N}), 8.25(\mathrm{~d}, 2 \mathrm{H}$, 
$J=8.8 \mathrm{~Hz}, \mathrm{Ar}-\mathrm{H}), 7.88(\mathrm{~d}, 2 \mathrm{H}, J=8.8 \mathrm{~Hz}, \mathrm{Ar}-\mathrm{H}), 7.59-7.44(\mathrm{~m}, 3 \mathrm{H}, \mathrm{Ar}-\mathrm{H}), 7.35(\mathrm{dd}, 2 \mathrm{H}, J=7.7 \mathrm{~Hz}$, $J=1.9 \mathrm{~Hz}, \mathrm{Ar}-\mathrm{H}), 4.00\left(\mathrm{~s}, 2 \mathrm{H}, \mathrm{CH}_{2}\right) ;{ }^{13} \mathrm{C}-\mathrm{NMR}\left(\mathrm{CDCl}_{3}\right): \delta 171.6(\mathrm{C}), 166.9(\mathrm{C}), 156.2(\mathrm{CH})$, $148.9(\mathrm{C}), 140.0(\mathrm{C}), 134.3(\mathrm{C}), 129.4(2 \mathrm{CH}), 129.3(\mathrm{CH}), 128.6(2 \mathrm{CH}), 127.7(2 \mathrm{CH}), 124.0(2 \mathrm{CH})$, $32.55\left(\mathrm{CH}_{2}\right)$; Anal. Calcd for $\mathrm{C}_{16} \mathrm{H}_{12} \mathrm{~N}_{4} \mathrm{O}_{3} \mathrm{~S}: \mathrm{C}, 56.46 ; \mathrm{H}, 3.55 ; \mathrm{N}, 16.46 ; \mathrm{S}, 9.42$. Found: C, 56.44; H, $3.5 ; \mathrm{N}, 16.53 ; \mathrm{S}, 9.53$.

2-((2,4-Dinitrobenzylidene)hydrazono)-3-phenylthiazolidin-4-one (6b): yellow solid; mp: $275{ }^{\circ} \mathrm{C}$; IR $(\mathrm{KBr}) v \max / \mathrm{cm}^{-1}: 1731(\mathrm{C}=\mathrm{O}), 1592(\mathrm{C}=\mathrm{N})$; ${ }^{1} \mathrm{H}-\mathrm{NMR}\left(\mathrm{DMSO}-\mathrm{d}_{6}\right): \delta 8.74(\mathrm{~d}, 1 \mathrm{H}, J=2.3 \mathrm{~Hz}$, Ar-H), $8.58(\mathrm{dd}, 1 \mathrm{H}, J=8.4 \mathrm{~Hz}, J=2.3 \mathrm{~Hz}, \mathrm{Ar}-\mathrm{H}), 8.57(\mathrm{~s}, 1 \mathrm{H}, \mathrm{HC}=\mathrm{N}), 8.20$ (d, $1 \mathrm{H}, J=8.4 \mathrm{~Hz}$, Ar-H), 7.54-7.45 (m, 3H, Ar-H), 7.41-7.36 (m, 2H, Ar-H), 4.14 (s, 2H, $\mathrm{CH}_{2}$ ); ${ }^{13} \mathrm{C}-\mathrm{NMR}$ (DMSO-d 6 ): $\delta 172.6(\mathrm{C}), 170.1(\mathrm{C}), 152.8(\mathrm{CH}), 148.4(\mathrm{C}), 148.3(\mathrm{C}), 135.3(\mathrm{C}), 133.7(\mathrm{C}), 131.2(\mathrm{CH}), 129.4$ $(2 \mathrm{CH}), 129.3(\mathrm{CH}), 128.7(2 \mathrm{CH}), 128.1(\mathrm{CH}), 120.6(\mathrm{CH}), 33.1\left(\mathrm{CH}_{2}\right)$; Anal. Calcd for $\mathrm{C}_{16} \mathrm{H}_{11} \mathrm{~N}_{5} \mathrm{O}_{5} \mathrm{~S}$ : C, 49.87; H, 2.88; N, 18.17; S, 8.32.Found: C, 49.99; H, 2.87; N, 17.93; S, 8.25.

2-(3-(2-Nitrophenyl)allylidene)hydrazono)-3-phenylthiazolidin-4-one (6c): orange solid; $\mathrm{mp}: 265{ }^{\circ} \mathrm{C}$; IR $(\mathrm{KBr}) v_{\max } / \mathrm{cm}^{-1}: 1732(\mathrm{C}=\mathrm{O}), 1627(\mathrm{C}=\mathrm{N}) ;{ }^{1} \mathrm{H}-\mathrm{NMR}\left(\mathrm{CDCl}_{3}\right): \delta 8.13(\mathrm{~d}, 1 \mathrm{H}, J=9.5 \mathrm{~Hz}, \mathrm{CH}=\mathrm{N}), 8.0$ (dd, $1 \mathrm{H}, J=8.2 \mathrm{~Hz}, J=1.0 \mathrm{~Hz}, \operatorname{Ar}-\mathrm{H}), 7.75-7.57$ (m, 3H, Ar-H), 7.53-7.43 (m, 4H, Ar-H), 7.39-7.32 (m, 2H, Ar-H), $7.01(\mathrm{dd}, 1 \mathrm{H}, J=15.8 \mathrm{~Hz}, J=9.5 \mathrm{~Hz}, \mathrm{CH}), 3.98\left(\mathrm{~s}, 2 \mathrm{H}, \mathrm{CH}_{2}\right) ;{ }^{13} \mathrm{C}-\mathrm{NMR}$ $\left(\mathrm{CDCl}_{3}\right): \delta 171.6(\mathrm{C}), 164.5(\mathrm{C}), 160.2(\mathrm{CH}), 148.00(\mathrm{C}), 135.8(\mathrm{CH}), 134.4(\mathrm{C}), 133.3(2 \mathrm{CH})$, $131.4(\mathrm{C}), 130.1(\mathrm{CH}), 129.4(2 \mathrm{CH}), 129.1(\mathrm{CH}), 128.3(\mathrm{CH}), 127.7(2 \mathrm{CH}), 125.0(\mathrm{CH}), 32.5\left(\mathrm{CH}_{2}\right)$; Anal. Calcd for $\mathrm{C}_{18} \mathrm{H}_{14} \mathrm{~N}_{4} \mathrm{O}_{3} \mathrm{~S}$ : C, 59.01; H, 3.85; N, 15.29; S, 8.75. Found: C, 58.96; H, 3.92; N, 15.18; S, 8.50.

2-(3,4-(Methylenedioxy)-6-nitrobenzaldehyde)hydrazono)-3-phenylthiazolidin-4-one (6d): yellow solid; mp: $262{ }^{\circ} \mathrm{C}$; IR $(\mathrm{KBr}) v \max / \mathrm{cm}^{-1}: 1717(\mathrm{C}=\mathrm{O}), 1602(\mathrm{C}=\mathrm{N}) ;{ }^{1} \mathrm{H}-\mathrm{NMR}\left(\mathrm{DMSO}-\mathrm{d}_{6}\right): \delta 8.47$ (s, $1 \mathrm{H}, \operatorname{Ar}-\mathrm{H}), 7.64(\mathrm{~s}, 1 \mathrm{H}, \mathrm{HC}=\mathrm{N}), 7.56-7.44(\mathrm{~m}, 3 \mathrm{H}, \mathrm{Ar}-\mathrm{H}), 7.35-7.40(\mathrm{~m}, 1 \mathrm{H}, \mathrm{Ar}-\mathrm{H}), 7.36(\mathrm{~m}, 2 \mathrm{H}$, $\mathrm{Ar}-\mathrm{H}), 6.27\left(\mathrm{~s}, 2 \mathrm{H}, \mathrm{CH}_{2}\right), 4.10\left(\mathrm{~s}, 2 \mathrm{H}, \mathrm{CH}_{2}\right) ;{ }^{13} \mathrm{C}-\mathrm{NMR}\left(\mathrm{DMSO}_{6}\right)$ : $\delta 172.5(\mathrm{C}), 167.7(\mathrm{C}), 154.0$ $(\mathrm{CH}), 152.0(\mathrm{C}), 149.8(\mathrm{C}), 144.0(\mathrm{C}), 135.4(\mathrm{C}), 129.6(2 \mathrm{CH}), 129.3(\mathrm{CH}), 128.7(2 \mathrm{CH}), 125.4(\mathrm{C})$, 106.6 (CH), $105.6(\mathrm{CH}), 104.4\left(\mathrm{CH}_{2}\right), 32.9\left(\mathrm{CH}_{2}\right)$; Anal. Calcd for $\mathrm{C}_{17} \mathrm{H}_{12} \mathrm{~N}_{4} \mathrm{O}_{5} \mathrm{~S}: \mathrm{C}, 53.12 ; \mathrm{H}, 3.15 ; \mathrm{N}$, 14.58; S, 8.34. Found: C, 53.15; H, 3.10; N, 14.67; S, 8.26.

2-((3,4,5-Trimethoxybenzylidene)hydrazono)-3-phenylthiazolidin-4-one (6e): white solid; mp: $174{ }^{\circ} \mathrm{C}$; IR $(\mathrm{KBr}) v \max / \mathrm{cm}^{-1}: 1720(\mathrm{C}=\mathrm{O}), 1619(\mathrm{C}=\mathrm{N}) ;{ }^{1} \mathrm{H}-\mathrm{NMR}\left(\mathrm{DMSO}-\mathrm{d}_{6}\right): \delta 8.21(\mathrm{~s}, 1 \mathrm{H}, \mathrm{HC}=\mathrm{N})$, 7.55-7.44 (m, 3H, Ar-H), 7.39-7.35 (m, 2H, Ar-H), 7.06 (s, 2H, Ar-H), 4.08 (s, 2H, $\left.\mathrm{CH}_{2}\right), 3.78$ (s, $\left.6 \mathrm{H}, \mathrm{OCH}_{3}\right), 3.70\left(\mathrm{~s}, 3 \mathrm{H}, \mathrm{OCH}_{3}\right) ;{ }^{13} \mathrm{C}-\mathrm{NMR}\left(\mathrm{DMSO}_{-} \mathrm{d}_{6}\right): \delta 172.6(\mathrm{C}), 165.4(\mathrm{C}), 158.0(\mathrm{CH}), 153.6$ (2C), $140.3(\mathrm{C}), 135.5(\mathrm{C}), 130.0(\mathrm{C}), 129.5(2 \mathrm{CH}), 129.1(\mathrm{CH}), 128.7(2 \mathrm{CH}), 105.5(2 \mathrm{CH}), 60.6$ $\left(\mathrm{CH}_{3}\right), 56.3\left(2 \mathrm{CH}_{3}\right), 32.7\left(\mathrm{CH}_{2}\right)$; Anal. Calcd for $\mathrm{C}_{19} \mathrm{H}_{19} \mathrm{~N}_{3} \mathrm{O}_{4} \mathrm{~S}: \mathrm{C}, 59.21 ; \mathrm{H}, 4.97 ; \mathrm{N}, 10.90 ; \mathrm{S}, 8.32$. Found: C, 59.29; H, 5.08; N, 10.64; S, 8.21.

2-(4-(Methylsulfonyl)benzylidene)hydrazono)-3-phenylthiazolidin-4-one (6f): yellow solid; mp: $277{ }^{\circ} \mathrm{C}$; IR (KBr) $v_{\max } / \mathrm{cm}^{-1}: 1734(\mathrm{C}=\mathrm{O}), 1615(\mathrm{C}=\mathrm{N}) ;{ }^{1} \mathrm{H}-\mathrm{NMR}\left(\mathrm{DMSO}-\mathrm{d}_{6}\right): \delta 8.43(\mathrm{~s}, 1 \mathrm{H}, \mathrm{HC}=\mathrm{N})$, 8.00 (d, 2H, $J=9.2 \mathrm{~Hz}, \mathrm{Ar}-\mathrm{H}), 7.94$ (d, 2H, $J=9.2 \mathrm{~Hz}, \mathrm{Ar}-\mathrm{H}), 7.54-7.44$ (m, 3H, Ar-H), 7.41-7.36 (m, 2H, Ar-H), $4.12\left(\mathrm{~s}, 2 \mathrm{H}, \mathrm{CH}_{2}\right), 3.22\left(\mathrm{~s}, 3 \mathrm{H}, \mathrm{CH}_{3}\right) ;{ }^{13} \mathrm{C}-\mathrm{NMR}\left(\mathrm{DMSO}-\mathrm{d}_{6}\right): \delta 172.5(\mathrm{C}), 167.8(\mathrm{C})$, 
$156.76(\mathrm{CH}), 142.5(\mathrm{C}), 139.2(\mathrm{C}), 135.4(\mathrm{C}), 129.6(2 \mathrm{CH}), 129.2(\mathrm{CH}), 128.7(4 \mathrm{CH}), 128.0(2 \mathrm{CH})$, $43.8\left(\mathrm{CH}_{3}\right), 32.9\left(\mathrm{CH}_{2}\right)$; Anal. Calcd for $\mathrm{C}_{17} \mathrm{H}_{15} \mathrm{~N}_{3} \mathrm{O}_{3} \mathrm{~S}_{2}: \mathrm{C}, 54.67 ; \mathrm{H}, 4.05 ; \mathrm{N}, 11.25 ; \mathrm{S}, 17.17$. Found: C, 54.71; H, 4.05; N, 11.19; S, 17.14 .

2-(4-(Methylthio)benzylidene)hydrazono)-3-phenylthiazolidin-4-one (6g): yellow solid; mp: $223{ }^{\circ} \mathrm{C}$; IR (KBr) $v_{\max } / \mathrm{cm}^{-1}: 1732(\mathrm{C}=\mathrm{O}), 1609(\mathrm{C}=\mathrm{N}) ;{ }^{1} \mathrm{H}-\mathrm{NMR}\left(\mathrm{CDCl}_{3}\right): \delta 8.25(\mathrm{~s}, 1 \mathrm{H}, \mathrm{HC}=\mathrm{N}), 7.64(\mathrm{~d}, 2 \mathrm{H}$, $J=8.5 \mathrm{~Hz}, \mathrm{Ar}-\mathrm{H}), 7.53-7.45$ (m, 3H, Ar-H), 7.38-7.34 (m, 2H, Ar-H), 7.21 (d, 2H, J=8.5 Hz, Ar-H), $3.97\left(\mathrm{~s}, 2 \mathrm{H}, \mathrm{CH}_{2}\right), 2.50\left(\mathrm{~s}, 3 \mathrm{H}, \mathrm{CH}_{3}\right) ;{ }^{13} \mathrm{C}-\mathrm{NMR}\left(\mathrm{CDCl}_{3}\right): \delta 171.8(\mathrm{C}), 163.9(\mathrm{C}), 158.4(\mathrm{CH}), 142.6$ (C), $134.5(\mathrm{C}), 130.7(\mathrm{C}), 129.4(2 \mathrm{CH}), 129.1(\mathrm{CH}), 128.4(2 \mathrm{CH}), 127.8(2 \mathrm{CH}), 125.8(2 \mathrm{CH}), 32.5$ $\left(\mathrm{CH}_{2}\right), 15.2\left(\mathrm{CH}_{3}\right)$; Anal. Calcd for $\mathrm{C}_{17} \mathrm{H}_{15} \mathrm{~N}_{3} \mathrm{OS}_{2}$ : C, 59.80; H, 4.43; N, 12.31; S, 18.78. Found: C, 59.67; H, 4.43; N, 12.20; S, 18.88 .

2-(4-(Trifluoromethyl)benzylidene)hydrazono)-3-phenylthiazolidin-4-one (6h): white solid; mp: $206{ }^{\circ} \mathrm{C}$; IR $(\mathrm{KBr}) v_{\max } / \mathrm{cm}^{-1}: 1732(\mathrm{C}=\mathrm{O}), 1616(\mathrm{C}=\mathrm{N}) ;{ }^{1} \mathrm{H}-\mathrm{NMR}\left(\mathrm{CDCl}_{3}\right): \delta 8.33(\mathrm{~s}, 1 \mathrm{H}, \mathrm{HC}=\mathrm{N})$, $7.84(\mathrm{~d}, 2 \mathrm{H}, J=8.2 \mathrm{~Hz}, \mathrm{Ar}-\mathrm{H}), 7.65$ (d, 2H, $J=8.2 \mathrm{~Hz}, \mathrm{Ar}-\mathrm{H}), 7.59-7.43$ (m, 3H, Ar-H), 7.39-7.32 (m, 2H, Ar-H), $3.99\left(\mathrm{~s}, 2 \mathrm{H}, \mathrm{CH}_{2}\right) ;{ }^{13} \mathrm{C}-\mathrm{NMR}\left(\mathrm{CDCl}_{3}\right)$ : $\delta 171.7(\mathrm{C}), 165.8(\mathrm{C}), 157.3(\mathrm{CH}), 137.4(\mathrm{C})$, $134.4(\mathrm{C}), 132(\mathrm{q}, J=32.4 \mathrm{~Hz}, \mathrm{C}), 129.4(2 \mathrm{CH}), 129.2(\mathrm{CH}), 128.2(2 \mathrm{CH}), 127.8(3 \mathrm{CH}), 125.6(\mathrm{q}$, $J=3.8 \mathrm{~Hz}, \mathrm{CH}), 123.8$ (q, $J=272.3 \mathrm{~Hz}, \mathrm{C}), 32.5\left(\mathrm{CH}_{2}\right)$; Anal. Calcd for $\mathrm{C}_{17} \mathrm{H}_{12} \mathrm{~F}_{3} \mathrm{~N}_{3} \mathrm{OS}: \mathrm{C}, 56.19$; $\mathrm{H}$, 3.33; N, 11.56; S, 8.82.Found: C, 56.17; H, 3.66; N, 12.82; S, 9.96.

\subsection{General Procedure for the Preparation of Compounds $\mathbf{8 a}-\mathbf{h}$}

An equimolar mixture of $\mathbf{4 a}-\mathbf{h}(1.5 \mathrm{mmol})$ and diethyl acetylenedicarboxylate $(1.5 \mathrm{mmol})$ in methanol $(20 \mathrm{~mL})$ was refluxed for $1 \mathrm{~h}$. After completion of the reaction, the reaction mixture was allowed to cool to the room temperature. The solid thus separated was collected by filtration and recrystallized using ethanol-DMF mixture.

(Z)-Ethyl-2-(2-((4-nitrobenzylidene)hydrazono)-4-oxo-3-phenylthiazolidin-5-ylidene) acetate (8a): yellow solid; mp: $206{ }^{\circ} \mathrm{C}$; IR $(\mathrm{KBr}) v_{\max } / \mathrm{cm}^{-1}$ : $1730(\mathrm{C}=\mathrm{O}), 1692(\mathrm{C}=\mathrm{O}), 1623(\mathrm{C}=\mathrm{N})$; ${ }^{1} \mathrm{H}-\mathrm{NMR}$ $\left(\mathrm{DMSO}_{-} \mathrm{d}_{6}\right): \delta 8.60(\mathrm{~s}, 1 \mathrm{H}, \mathrm{CH}=\mathrm{N}), 8.32(\mathrm{~d}, 2 \mathrm{H}, J=8.8 \mathrm{~Hz}, \mathrm{Ar}-\mathrm{H}), 8.01(\mathrm{~d}, 2 \mathrm{H}, J=8.8 \mathrm{~Hz}, \mathrm{Ar}-\mathrm{H})$, 7.58-7.48 (m, 5H, Ar-H), $6.80(\mathrm{~s}, 1 \mathrm{H}, \mathrm{C}=\mathrm{CH}), 4.28\left(\mathrm{q}, 2 \mathrm{H}, J=7.0 \mathrm{~Hz}, \mathrm{CH}_{2}\right), 1.28(\mathrm{t}, 3 \mathrm{H}, J=7.0 \mathrm{~Hz}$, $\left.\mathrm{CH}_{3}\right) .{ }^{13} \mathrm{C}-\mathrm{NMR}\left(\mathrm{DMSO}_{6}\right): \delta 166.0(\mathrm{C}), 164.6(\mathrm{C}), 163.6(\mathrm{C}), 158.5(\mathrm{CH}), 149.2(\mathrm{C}), 141.7(\mathrm{C})$, $140.0(\mathrm{C}), 134.5(\mathrm{C}), 129.6(3 \mathrm{CH}), 129.5(2 \mathrm{CH}), 128.6(2 \mathrm{CH}), 124.6(2 \mathrm{CH}), 116.2(\mathrm{CH}), 62.0\left(\mathrm{CH}_{2}\right)$, $14.5\left(\mathrm{CH}_{3}\right)$; Anal. Calcd for $\mathrm{C}_{20} \mathrm{H}_{16} \mathrm{~N}_{4} \mathrm{O}_{5} \mathrm{~S}: \mathrm{C}, 56.60 ; \mathrm{H}, 3.80 ; \mathrm{N}, 13.20 ; \mathrm{S}, 7.55$. Found: C, 55.68; H, $4.59 ; \mathrm{N}, 10.67 ; \mathrm{S}, 5.44$.

(Z)-Ethyl-2-(2-((E)-2,4-dinitrobenzylideneamino)-4-oxo-3-phenylthiazolidin-5-ylidene) acetate (8b): yellow solid; mp: $193{ }^{\circ} \mathrm{C}$; IR (KBr) $v_{\max } / \mathrm{cm}^{-1}$ : $1727(\mathrm{C}=\mathrm{O}), 1698(\mathrm{C}=\mathrm{O}), 1620(\mathrm{C}=\mathrm{N})$; ${ }^{1} \mathrm{H}-\mathrm{NMR}$ $\left(\mathrm{DMSO}_{-} \mathrm{d}_{6}\right): \delta 8.77(\mathrm{~d}, 1 \mathrm{H}, J=2.2 \mathrm{~Hz}, \mathrm{Ar}-\mathrm{H}), 8.7(\mathrm{~s}, 1 \mathrm{H}, \mathrm{CH}=\mathrm{N}), 8.64(\mathrm{dd}, 1 \mathrm{H}, J=8.6 \mathrm{~Hz}, J=2.2 \mathrm{~Hz}$, Ar-H), 8.25 (d, 1H, $J=8.6 \mathrm{~Hz}, \mathrm{Ar}-\mathrm{H}), 7.60-7.46(\mathrm{~m}, 5 \mathrm{H}, \mathrm{Ar}-\mathrm{H}), 6.83$ (s, 1H, C=CH), 4.29 (q, 2H, $\left.J=7.1 \mathrm{~Hz}, \mathrm{CH}_{2}\right), 1.28\left(\mathrm{t}, 3 \mathrm{H}, J=7.1 \mathrm{~Hz}, \mathrm{CH}_{3}\right) ;{ }^{13} \mathrm{C}-\mathrm{NMR}\left(\mathrm{DMSO}_{6}\right)$ ): $\delta 165.9(\mathrm{C}), 165.2(\mathrm{C}), 164.7$ (C), $155.2(\mathrm{CH}), 148.6(\mathrm{C}), 141.5(\mathrm{C}), 134.5(\mathrm{C}), 133.3(\mathrm{C}), 131.4(\mathrm{CH}), 129.8(\mathrm{CH}), 129.7(3 \mathrm{CH})$, 
$128.7(2 \mathrm{CH}), 128.3(\mathrm{CH}), 120.7(\mathrm{CH}), 116.6(\mathrm{CH}), 62.1\left(\mathrm{CH}_{2}\right), 14.5\left(\mathrm{CH}_{3}\right)$; Anal. Calcd for $\mathrm{C}_{20} \mathrm{H}_{15} \mathrm{~N}_{5} \mathrm{O}_{7} \mathrm{~S}$ : C, 51.17; H, 3.22; N, 14.92; S, 6.83. Found: C, 51.29; H, 3.15; N, 14.78; S, 6.77.

(Z)-Ethyl-2-(2-((E)-3-(2-nitrophenyl)allylidene)amino)-4-oxo-3-phenylthiazolidin-5-ylidene) acetate (8c): yellow solid; mp: $251{ }^{\circ} \mathrm{C}$; IR (KBr) $v_{\max } / \mathrm{cm}^{-1}$ : $1727(\mathrm{C}=\mathrm{O}), 1692(\mathrm{C}=\mathrm{O}), 1618(\mathrm{C}=\mathrm{N})$; ${ }^{1} \mathrm{H}-\mathrm{NMR}$ $\left(\mathrm{DMSO}_{-} \mathrm{d}_{6}\right): \delta 8.27(\mathrm{~d}, 1 \mathrm{H}, J=9.4 \mathrm{~Hz}, \mathrm{CH}), 8.01(\mathrm{ddd}, 2 \mathrm{H}, J=7.1 \mathrm{~Hz}, J=7.9 \mathrm{~Hz}$, $J=1.1 \mathrm{~Hz}, \mathrm{Ar}-\mathrm{H}), 7.73$ (dd, 1H, $J=7.9 \mathrm{~Hz}, J=7.1 \mathrm{~Hz}, \mathrm{Ar}-\mathrm{H}), 7.63-7.42$ (m, 7H, Ar-H), 7.20 (dd, 1H, $J=15.7 \mathrm{~Hz}, J=9.4 \mathrm{~Hz}, \operatorname{Ar}-\mathrm{H}), 6.77(\mathrm{~s}, 1 \mathrm{H}, \mathrm{C}=\mathrm{CH}), 4.27\left(\mathrm{q}, 2 \mathrm{H}, J=7.1 \mathrm{~Hz}, \mathrm{CH}_{2}\right), 1.27(\mathrm{t}, 3 \mathrm{H}$, $\left.J=7.1 \mathrm{~Hz}, \mathrm{CH}_{3}\right) ;{ }^{13} \mathrm{C}-\mathrm{NMR}\left(\mathrm{DMSO}_{6}\right): \delta 166.0(\mathrm{C}), 164.6(\mathrm{C}), 162.1(\mathrm{CH}), 161.5(\mathrm{C}), 148.6(\mathrm{C})$, $141.9(\mathrm{C}), 137.3(\mathrm{CH}), 134.6(\mathrm{C}), 134.0(\mathrm{CH}), 130.7(\mathrm{CH}), 130.4(\mathrm{C}), 129.8(\mathrm{CH}), 129.6(3 \mathrm{CH})$, $129.0(\mathrm{CH}), 128.6(2 \mathrm{CH}), 125.0(\mathrm{CH}), 115.9(\mathrm{CH}), 62.0\left(\mathrm{CH}_{2}\right), 14.5\left(\mathrm{CH}_{3}\right)$. Anal. Calcd for $\mathrm{C}_{22} \mathrm{H}_{18} \mathrm{~N}_{4} \mathrm{O}_{5} \mathrm{~S}$ : C, 58.66; H, 4.03; N, 12.44; S, 7.12. Found: C, 58.47; H, 3.92; N, 12.05; S, 6.64

(Z)-Ethyl-2-(2-((E)-(4-nitrobenzo[d] [1,3]dioxol-5-yl)methyleneamino)-4-oxo-3-phenylthiazolidin-5ylidene) acetate (8d): yellow solid; mp: $251{ }^{\circ} \mathrm{C}$; IR $(\mathrm{KBr}) v_{\max } / \mathrm{cm}^{-1}: 1721(\mathrm{C}=\mathrm{O}) 1702(\mathrm{C}=\mathrm{O}), 1597$ $(\mathrm{C}=\mathrm{N}) ;{ }^{1} \mathrm{H}-\mathrm{NMR}\left(\mathrm{DMSO}_{6}\right): \delta 8.59$ (s, 1H, Ar-H), 7.70 (s, 1H, Ar-H), 7.54-7.47 (m, 5H, Ar-H), 7.38 (s, 1H, Ar-H), $6.80(\mathrm{~s}, 1 \mathrm{H}, \mathrm{C}=\mathrm{CH}), 6.30\left(\mathrm{~s}, 2 \mathrm{H}, \mathrm{CH}_{2}\right), 4.27$ (q, 2H, J=7.1 Hz, CH ), 1.27 (t, 3H, $\left.J=7.1 \mathrm{~Hz}, \mathrm{CH}_{3}\right) ;{ }^{13} \mathrm{C}-\mathrm{NMR}\left(\mathrm{DMSO}_{\mathrm{d}}\right): \delta 166.0(\mathrm{C}), 164.7(\mathrm{C}), 163.0(\mathrm{C}), 156.5(\mathrm{CH}), 152.1(\mathrm{C})$, $150.3(\mathrm{C}), 144.3(\mathrm{C}), 141.8(\mathrm{C}), 137.2(\mathrm{C}), 134.6(\mathrm{C}), 129.7(2 \mathrm{CH}), 128.7(2 \mathrm{CH}), 124.9(\mathrm{CH}), 116.2$ $(\mathrm{CH}), 106.8(\mathrm{CH}), 105.8(\mathrm{CH}), 104.6\left(\mathrm{CH}_{2}\right), 62.0\left(\mathrm{CH}_{2}\right), 14.5\left(\mathrm{CH}_{3}\right)$; Anal. Calcd for $\mathrm{C}_{21} \mathrm{H}_{16} \mathrm{~N}_{4} \mathrm{O}_{7} \mathrm{~S}: \mathrm{C}$, 53.84; H, 3.44; N, 11.96; S, 6.85. Found: C, 53.79; H, 3.42; N, 11.98; S, 6.75.

(Z)-Ethyl-2-(2-((E)-3,4,5-trimethoxybenzylideneamino)-4-oxo-3-phenylthiazolidin-5-ylidene) acetate (8e): yellow solid; $\mathrm{mp}: 145{ }^{\circ} \mathrm{C}$; IR (KBr) $v_{\max } / \mathrm{cm}^{-1}$ : $1721(\mathrm{C}=\mathrm{O}), 1689(\mathrm{C}=\mathrm{O}), 1622(\mathrm{C}=\mathrm{N})$; ${ }^{1} \mathrm{H}-\mathrm{NMR}$ $\left(\mathrm{DMSO}_{\mathrm{d}}\right): \delta 8.35(\mathrm{~s}, 1 \mathrm{H}, \mathrm{CH}=\mathrm{N}), 7.58-7.47(\mathrm{~m}, 5 \mathrm{H}, \mathrm{Ar}-\mathrm{H}), 7.11(\mathrm{~s}, 2 \mathrm{H}, \mathrm{Ar}-\mathrm{H}), 6.77$ $(\mathrm{s}, 1 \mathrm{H}, \mathrm{C}=\mathrm{CH}), 4.27$ (q, 2H, $\left.J=7.0 \mathrm{~Hz}, \mathrm{CH}_{2}\right), 3.80\left(\mathrm{~s}, 6 \mathrm{H}, \mathrm{OCH}_{3}\right), 3.70\left(\mathrm{~s}, 3 \mathrm{H}, \mathrm{OCH}_{3}\right), 1.28(\mathrm{t}, 3 \mathrm{H}$, $\left.J=7.0 \mathrm{~Hz}, \mathrm{CH}_{3}\right) ;{ }^{13} \mathrm{C}-\mathrm{NMR}\left(\mathrm{DMSO}_{6}\right)$ : $\delta 165.9(\mathrm{C}), 164.6(\mathrm{C}), 160.6(\mathrm{C}), 160.4(\mathrm{CH}), 153.6(2 \mathrm{C})$, 142.0 (C), $140.9(\mathrm{C}), 134.7(\mathrm{C}), 129.6(3 \mathrm{CH}), 129.4(\mathrm{C}), 128.6(2 \mathrm{CH}), 115.7(\mathrm{CH}), 105.9(2 \mathrm{CH}), 62.0$ $\left(\mathrm{CH}_{2}\right), 60.6\left(\mathrm{CH}_{3}\right), 56.3\left(2 \mathrm{CH}_{3}\right), 14.5\left(\mathrm{CH}_{3}\right)$; Anal. Calcd for $\mathrm{C}_{23} \mathrm{H}_{23} \mathrm{~N}_{3} \mathrm{O}$ 6 $\mathrm{S}: \mathrm{C}, 58.84 ; \mathrm{H}, 4.94 ; \mathrm{N}, 8.95$; S, 6.83. Found: C, 58.47; H, 4.93; N, 8.77; S, 6.64 .

(Z)-Ethyl-2-(2-((E)-4-(methylsulfonyl)benzylideneamino)-4-oxo-3-phenylthiazolidin-5-ylidene) acetate (8f): yellow solid; mp: $254{ }^{\circ} \mathrm{C}$; IR $(\mathrm{KBr}) \operatorname{vmax} / \mathrm{cm}^{-1}$ : $1722(\mathrm{C}=\mathrm{O}), 1687(\mathrm{C}=\mathrm{O}), 1611(\mathrm{C}=\mathrm{N})$; ${ }^{1} \mathrm{H}-\mathrm{NMR}\left(\mathrm{DMSO}_{\mathrm{d}}\right): \delta 8.58(\mathrm{~s}, 1 \mathrm{H}, \mathrm{CH}=\mathrm{N}), 8.02(\mathrm{~s}, 4 \mathrm{H}, \mathrm{Ar}-\mathrm{H}), 7.59-7.45(\mathrm{~m}, 5 \mathrm{H}, \mathrm{Ar}-\mathrm{H}), 6.81$ (s, $1 \mathrm{H}, \mathrm{C}=\mathrm{CH}), 4.29\left(\mathrm{q}, 2 \mathrm{H}, J=7.1 \mathrm{~Hz}, \mathrm{CH}_{2}\right), 3.24\left(\mathrm{~s}, 3 \mathrm{H}, \mathrm{CH}_{3}\right), 1.28\left(\mathrm{t}, 3 \mathrm{H}, J=7.1 \mathrm{~Hz}, \mathrm{CH}_{3}\right) ;{ }^{13} \mathrm{C}-\mathrm{NMR}$ $\left(\mathrm{DMSO}_{\mathrm{d}}\right.$ ): $\delta 166.0(\mathrm{C}), 164.7(\mathrm{C}), 163.2(\mathrm{C}), 159.0(\mathrm{CH}), 143.0(\mathrm{C}), 141.8(\mathrm{C}), 138.6(\mathrm{C}), 134.6(\mathrm{C})$, $129.7(3 \mathrm{CH}), 129.1(2 \mathrm{CH}), 128.7(2 \mathrm{CH}), 128.1(2 \mathrm{CH}), 116.1(\mathrm{CH}), 62.1\left(\mathrm{CH}_{2}\right), 43.9\left(\mathrm{CH}_{3}\right), 14.5$ $\left(\mathrm{CH}_{3}\right)$; Anal. Calcd for $\mathrm{C}_{21} \mathrm{H}_{19} \mathrm{~N}_{3} \mathrm{O}_{5} \mathrm{~S}_{2}$ : C, 55.13; H, 4.19; N 9.18; S, 14.02. Found: C, 55.18; H, 4.19; N, 8.96; S, 14.11 .

(Z)-Ethyl-2-(2-((E)-4-(methylthio)benzylideneamino)-4-oxo-3-phenylthiazolidin-5-ylidene)acetate

(8g): yellow solid; mp: $210{ }^{\circ} \mathrm{C}$; IR (KBr) v $v_{\max } / \mathrm{cm}^{-1}$ : $1720(\mathrm{C}=\mathrm{O}), 1696(\mathrm{C}=\mathrm{O}), 1604(\mathrm{C}=\mathrm{N})$; ${ }^{1} \mathrm{H}-\mathrm{NMR}$ $\left(\mathrm{DMSO}_{-} \mathrm{d}_{6}\right): \delta 8.40(\mathrm{~s}, 1 \mathrm{H}, \mathrm{CH}=\mathrm{N}), 8.70(\mathrm{~d}, 2 \mathrm{H}, J=8.4 \mathrm{~Hz}, \mathrm{Ar}-\mathrm{H}), 7.58-7.44(\mathrm{~m}, 5 \mathrm{H}, \mathrm{Ar}-\mathrm{H}), 7.33(\mathrm{~d}$, 
$2 \mathrm{H}, J=8.4 \mathrm{~Hz}, \mathrm{Ar}-\mathrm{H}), 6.77(\mathrm{~s}, 1 \mathrm{H}, \mathrm{C}=\mathrm{CH}), 4.28\left(\mathrm{q}, 2 \mathrm{H}, J=7.1 \mathrm{~Hz}, \mathrm{CH}_{2}\right), 3.31\left(\mathrm{~s}, 3 \mathrm{H}, \mathrm{CH}_{3}\right), 1.28$ (t, 3H, $J=7.1 \mathrm{~Hz}, \mathrm{CH}_{3}$ ); ${ }^{13} \mathrm{C}-\mathrm{NMR}$ (DMSO-d D ): $\delta 166.0(\mathrm{C}), 164.6(\mathrm{C}), 160.9(\mathrm{C}), 160.0(\mathrm{CH}), 143.5$ (C), $142.1(\mathrm{C}), 134.7(\mathrm{C}), 130.3(\mathrm{C}), 129.6(3 \mathrm{CH}), 128.9(2 \mathrm{CH}), 128.7(2 \mathrm{CH}), 126.0(2 \mathrm{CH}), 115.6$ (CH), $62.0\left(\mathrm{CH}_{2}\right), 14.6\left(\mathrm{CH}_{3}\right), 14.5\left(\mathrm{CH}_{3}\right)$; Anal. Calcd for $\mathrm{C}_{21} \mathrm{H}_{19} \mathrm{~N}_{3} \mathrm{O}_{3} \mathrm{~S}_{2}: \mathrm{C}, 59.27 ; \mathrm{H}, 4.50 ; \mathrm{N} 9.87$; S, 15.07. Found: C, 59.10; H, 4.48; N, 9.74; S, 14.86.

(Z)-Ethyl-2-(2-((E)-4-(trifluoromethyl)benzylideneamino)-4-oxo-3-phenylthiazolidin-5-ylidene) acetate (8h): yellow solid; mp: $154{ }^{\circ} \mathrm{C}$; IR $(\mathrm{KBr}) v_{\max } / \mathrm{cm}^{-1}$ : $1721(\mathrm{C}=\mathrm{O}), 1690(\mathrm{C}=\mathrm{O}), 1619(\mathrm{C}=\mathrm{N})$; ${ }^{1} \mathrm{H}-\mathrm{NMR}$ $\left(\mathrm{DMSO}_{6}\right): \delta 8.56(\mathrm{~s}, 1 \mathrm{H}, \mathrm{CH}=\mathrm{N}), 8.00(\mathrm{~d}, 2 \mathrm{H}, J=8.2 \mathrm{~Hz}, \mathrm{Ar}-\mathrm{H}), 7.84(\mathrm{~d}, 2 \mathrm{H}, J=8.2 \mathrm{~Hz}, \mathrm{Ar}-\mathrm{H})$, 7.58-7.45 (m, 5H, Ar-H), $6.80(\mathrm{~s}, 1 \mathrm{H}, \mathrm{C}=\mathrm{CH}), 4.30\left(\mathrm{q}, 2 \mathrm{H}, J=7.1 \mathrm{~Hz}, \mathrm{CH}_{2}\right), 1.28(\mathrm{t}, 3 \mathrm{H}$, $\left.J=7.1 \mathrm{~Hz}, \mathrm{CH}_{3}\right) ;{ }^{13} \mathrm{C}-\mathrm{NMR}\left(\mathrm{DMSO}_{6}\right): \delta 166.0(\mathrm{C}), 164.6(\mathrm{C}), 162.9(\mathrm{C}), 159.1(\mathrm{CH}), 141.9(\mathrm{C})$, 137.9 (C), 134.6 (C), 131.3 (q, $J=32.2 \mathrm{~Hz}, \mathrm{C}), 129.6$ (q, $J=272.2 \mathrm{~Hz}, \mathrm{C}), 129.5(3 \mathrm{CH}), 129.1(2 \mathrm{CH})$, $128.7(2 \mathrm{CH}), 126.3(\mathrm{q}, J=3.2 \mathrm{~Hz}, 2 \mathrm{CH}), 116.0(\mathrm{CH}), 62.0\left(\mathrm{CH}_{2}\right), 14.5\left(\mathrm{CH}_{3}\right)$; Anal. Calcd for $\mathrm{C}_{21} \mathrm{H}_{16} \mathrm{~F}_{3} \mathrm{~N}_{3} \mathrm{O}_{3} \mathrm{~S}$ : C, 56.37; H, 3.60; N, 9.39; S, 7.17. Found: C, 56.33; H, 3.51; N, 9.17; S, 7.10.

\section{Conclusions}

In conclusion, we have prepared a series of 4-phenyl-3-thiosemicarbazone derivatives from 4-phenyl-3-thiosemicarbazide and various aromatic aldehydes substituted with different electron-donor and -withdrawing groups. In a second step, these 4-phenyl-3-thiosemicarbazone derivatives were reacted with 2-ethyl bromoacetate and diethyl acetylenedicarboxylate, respectively, to afford an original series of highly functionalized thiazolidinone derivatives in good yields. The antiparasitic and antibacterial evaluations of all synthesized compound are under investigation.

\section{Acknowledgments}

This work was supported by the CNEPRU (Ministry of Higher Education and Scientific Research Algeria). We express our thanks to Vincent Remusat for recording the ${ }^{1} \mathrm{H}$ and ${ }^{13} \mathrm{C}-\mathrm{NMR}$ spectra.

\section{Author Contributions}

A.D.; T.T. and P.V. conceived and designed the study. A.B. and O.K. designed the experiments and interpreted the results. A.B.; O.K.; A.D.; T.T. and P.V. wrote the manuscript.

\section{Conflicts of Interest}

The authors declare no conflict of interest.

\section{References and Notes}

1. Pirrung, M.C.; Pansare, S.V.; das Sarma, K.; Keith, K.A.; Kern, E.R. Combinatorial optimization of isatin- $\beta$-thiosemicarbazones as anti-poxvirus agents. J. Med. Chem. 2005, 48, 3045-3050.

2. Hu, W.-X.; Zhou, W.; Xia, C.-N.; Wen, X. Synthesis and anticancer activity of thiosemicarbazones. Bioorg. Med. Chem. Lett. 2006, 16, 2213-2218. 
3. Kolocouris, A.; Dimas, K.; Pannecouque, C.; Witvrouw, M.; Foscolos, G.B.; Stamatiou, G.; Fytas, G.; Zoidis, G.; Kolocouris, N.; Andrei, G.; et al. New 2-(1-adamantylcarbonyl) pyridine and 1-acetyladamantane thiosemicarbazones-thiocarbonohydrazones: Cell growth inhibitory, antiviral and antimicrobial activity evaluation. Bioorg. Med. Chem. Lett. 2002, 12, 723-727.

4. Tarasconi, P.; Capacchi, S.; Pelosi, G.; Cornia, M.; Albertini, R.; Bonati, A.; Dall'Aglio, P.P.; Lunghi, P.; Pinelli, S. Synthesis, spectroscopic characterization and biological properties of new natural aldehydes thiosemicarbazones. Bioorg. Med. Chem. 2000, 88, 157.

5. Pérez-Rebolledo, A.; Teixeira, L.R.; Batista, A.A.; Mangrich, A.S.; Aguirre, G.; Cerceretto, H.; González, M.; Hernández, P.; Ferreira, A.M.; Speziali, N.L.; et al. 4-Nitroacetophenone-derived thiosemicarbazones and their copper (II) complexes with significant in vitro anti-trypanosomal activity. Eur. J. Med. Chem. 2008, 43, 939-948.

6. Aguirre, G.; Boiani, L.; Cerecetto, H.; Fernández, M.; González, M.; Denicola, A.; Otero, L.; Gambino, D.; Rigol, C.; Olea-Azar, C.; et al. In vitro activity and mechanism of action against the protozoan parasite Trypanosoma cruzi of 5-nitrofuryl containing thiosemicarbazones. Bioorg. Med. Chem. 2004, 12, 4885-4893.

7. Du, X.; Guo, C.; Hansell, E.; Doyle, P.S.; Caffrey, C.R.; Holler, T.P.; McKerrow, J.H.; Cohen, E. Synthesis and structure-activity relationship study of potent trypanocidal thio- semicarbazone inhibitors of the trypanosomal cysteine protease cruzain. J. Med. Chem. 2002, 45, 2695-2707.

8. De Aquino, T.M.; Liesen, A.P.; da Silva, R.E.A.; Lima, V.T.; Carvalho, C.S.; de Faria, A.R.; de Araujo, J.M.; de Lima, J.G.; Alves, A.J.; de Melo, E.J.T.; et al. Synthesis, anti-Toxoplasma-gondii and antimicrobial activities of benzaldehyde 4-phenyl-3-thiosemicarbazones and 2-[(phenymethylene) hydrazono]-4-oxo-3-phenyl-5-thiazolidine acetic acids. Bioorg. Med. Chem. 2008, 16, 446-456.

9. Mayekar, S.A.; Mulwad, V.V. Synthesis and antibacterial activity of 6-(5phenyl- $\{1,3,4\}$ thiadiazol-2-ylimino)-benzopyran-2-ones. Ind. J. Chem. 2008, 47, 1438-1442.

10. Omar, K.; Geronikaki, A.; Zoumpoulakis, P.; Camoutsis, C.; Sokovic, M.; Ciric, A.; Glamoclija, J.; Novel 4- thiazolidinone derivatives as potential antifungal and antibacterial drugs. Bioorg. Med. Chem. 2010, 18, 426-432.

11. Bhat, M.A.; Siddiqui, N.; Khan, S.A. Synthesis, anticonvulsant and neurotoxicity screening of 2-(substituted phenyl)-3-[3-(2-oxo-2H-chromen-3-yl)-5-thioxo-1,5-dihydro-4H-1,2,4-triazol-4-yl]1,3-thiazolidin-4-ones. Ind. J. Het. Chem. 2008, 17, 287-288.

12. Babaoglu, K.; Page, M.A.; Jones, V.C.; McNeil, M.R.; Dong, C.; Naismith, J.H.; Lee, R.E. Novel inhibitors of an emerging target in mycobacterium tuberculosis; substituted thiazolidinones as inhibitors of dTDP-rhamnose synthesis. Bioorg. Med. Chem. Lett. 2003, 13, 3227-3230.

13. Vigorita, M.G.; Ottanà, R.; Monforte, F.; Maccari, R.; Monforte, M.T.; Trovato, A.; Taviano, M.F.; Miceli, N.; de Luca, G.; Alcaro, S.; et al. Chiral 3,3'-(1,2-ethanediyl)-bis[2-(3,4-dimethoxyphenyl)4-thiazolidinones] with anti-inflammatory activity. Part 11: Evaluation of COX-2 selectivity and modeling. Bioorg. Med. Chem. 2003, 11, 999-1006.

14. Agrawal, V.K.; Sachan, S.; Khadikar, P.V. QSAR studies on antihistaminic activity of some thiazolidine-4-ones. Acta Pharm. 2000, 50, 281-290.

15. Diurno, M.V.; Mazzoni, O.; Correale, G.; Monterrey, I.G.; Calignano, A.; la Rana, G.; Bolognese, A. Synthesis and structure-activity relationships of 2-(substituted phenyl)-3-[3-(N,N-dimethylamino)propyl]-1,3-thiazolidin-4-ones acting as H1-histamine antagonists. Farmaco 1999, 54, 579-583. 
16. Suzuki, Y.; Akima, M.; Tamura, K. Effects of CP-060S, a novel cardioprotective drug, on cardiac function and myocardial oxygen consumption. Gen. Pharmacol. 1999, 32, 57-63.

17. Rawal, R.K.; Prabhakar, Y.S.; Katti, S.B.; de Clercq, E. 2-(Aryl)-3-furan-2-ylmethyl-thiazolidin4-ones as selective HIV-RT inhibitors. Bioorg. Med. Chem. 2005, 13, 6771-6776.

18. Crozet, M.P.; Archaimbault, G.; Vanelle, P.; Nouguier, R. Reaction $\mathrm{S}_{\mathrm{RN}} 1$ en serie heterocyclique: Reactivite des sels du dimethyl-2,2-nitro-5-dioxane-1,3. Tetrahedron Lett. 1985, 26, 5133-5134.

19. Amiri-Attou, O.; Terme, T.; Vanelle, P. Functionalization of 6-nitrobenzo[1,3]dioxole with carbonyl compounds via TDAE methodology. Molecules 2005, 10, 545-551.

20. Verhaeghe, P.; Azas, N.; Hutter, S.; Castera-Ducros, C.; Laget, M.; Dumètre, A.; Gasquet, M.; Reboul, J.P.; Rault, S.; Rathelot, P.; et al. Synthesis and in vitro antiplasmodial evaluation of 4-anilino-2-trichloromethylquinazolines. Bioorg. Med. Chem. 2009, 17, 4313-4322.

21. Crozet, M.D.; Botta, C.; Gasquet, M.; Curti, C.; Remusat, V.; Hutter, S.; Chapelle, O.; Azas, N.; de Méo, M.; Vanelle, P. Lowering of 5-nitroimidazole's mutagenicity: Towards optimal antiparasitic pharmacophore. Eur. J. Med. Chem. 2009, 44, 653-659.

22. Kabri, Y.; Azas, N.; Dumètre, A.; Hutter, S.; Laget, M.; Verhaeghe, P.; Gellis, A.; Vanelle, P. Original quinazoline derivatives displaying antiplasmodial properties. Eur. J. Med. Chem. 2010, $45,616-622$.

23. Huang, H.; Chen, Q.; Ku, X.; Meng, L.; Lin, L.; Wang, X.; Zhu, C.; Wang, Y.; Chen, Z.; Li, M.; et al. A series of $\alpha$-heterocyclic carboxaldehyde thiosemicarbazones inhibit topoisomerase II $\alpha$ catalytic activity. J. Med. Chem. 2010, 53, 3048-3064.

24. Serda, M.; Malecki, J.G.; Mrozek-Wilczkiewicz, A.; Musiol, R.; Polanski, J. Microwave assisted synthesis, X-ray crystallography and DFT calculations of selected aromatic thiosemicarbazones. J. Mol. Struct. 2013, 1037, 63-72.

25. Küçügüzel, G.; Kocayepe, A.; de Clercq, E.; Sahin, F.; Güllüce, M. Synthesis and biological activity of 4-thiazolidinones, thiosemicarbazides derived from diflunisal hydrazide. Eur. J. Med. Chem. 2006, 41, 353-359.

26. Vas'kevich, R.I.; Zborovskii, Y.L.; Staninets, V.I.; Chernega, A.N. Reaction of 4-Aryl-1-(4-oxo3,4-dihydrothieno[2,3- $d$ ]pyrimidin-2-yl)thiosemicarbazides with Dimethyl Acetylenedicarboxylate. Russ. J. Org. Chem. 2004, 40, 1047-1052.

27. CCDC contains the supplementary crystallographic data of compound $\mathbf{8 b}$ for this paper. These data can be obtained free of charge from The Cambridge Crystallographic Data Centre via http://www.ccdc.cam.ac.uk/data_request/cif.

28. CCDC contains the supplementary crystallographic data of compound $\mathbf{8 g}$ for this paper. These data can be obtained free of charge from The Cambridge Crystallographic Data Centre via http://www.ccdc.cam.ac.uk/data_request/cif.

Sample Availability: Samples of the compounds $4 \mathbf{a}-\mathbf{h}, \mathbf{6} \mathbf{a}-\mathbf{h}$ and $\mathbf{8 a}-\mathbf{h}$ are available from the authors.

(C) 2014 by the authors; licensee MDPI, Basel, Switzerland. This article is an open access article distributed under the terms and conditions of the Creative Commons Attribution license (http://creativecommons.org/licenses/by/3.0/). 